TI 2013-208/II

Tinbergen Institute Discussion Paper

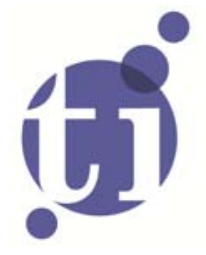

\title{
Diffusion of Behavior in Network Games orchestrated by Social Learning
}

Jia-Ping Huang

Maurice Koster ${ }^{2}$

Ines Lindnert,3

' Faculty of Economics and Business Administration, VU University Amsterdam;

2 Amsterdam School of Economics, University of Amsterdam;

3 Tinbergen Institute. 
Tinbergen Institute is the graduate school and research institute in economics of Erasmus University Rotterdam, the University of Amsterdam and VU University Amsterdam.

More TI discussion papers can be downloaded at http://www.tinbergen.nl

Tinbergen Institute has two locations:

Tinbergen Institute Amsterdam

Gustav Mahlerplein 117

1082 MS Amsterdam

The Netherlands

Tel.: +31(0)205251600

Tinbergen Institute Rotterdam

Burg. Oudlaan 50

3062 PA Rotterdam

The Netherlands

Tel.: +31(0)10 4088900

Fax: $+31(0) 104089031$

Duisenberg school of finance is a collaboration of the Dutch financial sector and universities, with the ambition to support innovative research and offer top quality academic education in core areas of finance.

DSF research papers can be downloaded at: http://www.dsf.nl/

Duisenberg school of finance

Gustav Mahlerplein 117

1082 MS Amsterdam

The Netherlands

Tel.: +31(0)20 5258579 


\title{
Diffusion of Behavior in Network Games Orchestrated by Social Learning
}

\author{
Jia-Ping Huang* ${ }^{*}$ Maurice Koster ${ }^{\dagger}$ Ines Lindner ${ }^{\ddagger}$
}

\begin{abstract}
The novelty of our model is to combine models of collective action on networks with models of social learning. Agents are connected according to an undirected graph, the social network, and have the choice between two actions: either to adopt a new behavior or technology or stay with the default behavior. The individual believed return depends on how many neighbors an agent has, how many of those neighbors already adopted the new behavior and some agent-specific cost-benefit parameter. There are four main insights of our model: (1) A variety of collective adoption behaviors is determined by the network. (2) Average inclination governs collective adoption behavior. (3) Initial inclinations determine the critical mass of adoption which ensures the new behavior to prevail. (4) Equilibria and dynamic behavior changes as we change the underlying network and other parameters. Given the complexity of the system we use a standard technique for estimating the solution.
\end{abstract}

Keywords: Diffusion, Social Networks, Social Learning, Tipping, Technology Adoption. JEL: C72; C73; D83; D85; O33.

\section{Introduction}

The interest in understanding the driving forces of collective behavior has triggered an extensive literature that spans economics, sociology, marketing and epidemiology. In

*Department of Econometrics \& OR, VU University Amsterdam, De Boelelaan 1105, 1081 HV Amsterdam, The Netherlands, email: j.huang@vu.nl

${ }^{\dagger}$ CeNDEF, Amsterdam School of Economics, University of Amsterdam, The Netherlands, email: m.a.l.koster@uva.nl

$\ddagger(i)$ Tinbergen Institute, Amsterdam

(ii) Department of Econometrics \& OR, VU University Amsterdam, De Boelelaan 1105, 1081 HV Amsterdam, The Netherlands, email: i.d.lindner@vu.nl 
their seminal work, Schelling [1971] and Granovetter [1978] develop models of collective behavior for situations where individuals have two alternatives and the costs and/or benefits of each depend on how many individuals choose which alternative. Examples are numerous, including riot behavior, innovation and rumor diffusion, strikes, consumption network externalities, spread of fashions, etc. The key element of Schelling [1971] and Granovetter [1978] is the concept of a threshold, i.e., the number or proportion of others who must take action before a given individual does so, or equivalently, the point at which net benefit begins to exceed net cost for that particular individual. Characteristic of these models is that a particular action alternative will only be adopted on a large scale if it achieves some critical mass of support. A major drawback of these models, however, is that they don't provide an explanation where this critical mass comes from as it is usually treated as an exogenous factor. In the context of diffusion of innovation Jackson and Yariv [2005, 2007] offer the metaphor of a free trial period of new technology. In his models of rioting behavior Kuran [1989, 1991] suggests that the early mobilization problem will only be resolved through a catalytic event, a spark, that reveals the hidden unpopularity of the present regime. On the outbreak of the revolutions in Eastern Europe and the French Revolution he notes, however, that this spark is often difficult to discern, explaining why these occurrences seem to "appear out of nowhere".

Another drawback of Schelling [1971] and Granovetter [1978] is that the structure of communication among individuals is not explicitly modeled; implicitly the seminal models assume that every individual is informed about the decision of everybody else. Jackson and Yariv [2005, 2007] showed how to extend Granovetter's model by introducing social networks to the framework. Agents are connected according to an undirected graph (the social network) and decide whether to switch to a new behavior, e.g. a new technology, or stay with the default alternative. The return to each action depends on the number of neighbors in the network, the proportion of neighbors adopting the new alternative and some agent-specific cost-benefit parameters. Individuals are supposed to be myopic and play best responses to the neighbors' action in the previous period. Similar to the classical works their model features the existence of a tipping point which is largely determined by the network. Although Jackson and Yariv successfully give an answer to the effect of social network structures the question remains about the endogenous triggers and driving forces of collective action. As noted in Valente [2005] in the context of diffusion of innovation, "Verbal accounts on how people make decisions and adopt behavior usually reveal ... whims that are not independent of networks, but not easily captured in social influence models." The state-of-the-art literature is not capable of explaining the hidden driving force of collective action as an endogenous property of dynamics.

In his overview article about innovation diffusion, Young [2009] compares three broad classes of models: contagion, social influence, and social learning. He summarizes the 
approach of social learning as "People adopt once they see enough empirical evidence to convince them that the innovation is worth adopting, where the evidence is generated by the outcomes among prior adopters. Individuals may adopt at different times due to differences in their prior beliefs, amount of information gathered, and idiosyncratic costs." Hence people want to see how it works for others over a period of time before trying themselves. As he points out it is difficult to summarize the sizable literature on social learning due to the great diversity of informational assumptions. He nevertheless identifies the dynamic characteristics of a fairly general class of learning models which has a surprisingly simple structure. It boils down to Bayesian updating of beliefs about the quality of innovation relative to the status quo. Individuals have initial beliefs about the payoff of the innovation, based on partial information, and update their beliefs through a random meeting process with adopters. The updated belief is the weighted average of initial belief and the average payoff of the observations. Individuals adopt the innovation when their updated beliefs exceed their costs. Effects of the social network architecture are not discussed as he implicitly assumes a complete communication network.

The novelty of our model is to combine models of collective action on networks with models of social learning. The model is therefore described by two dimensions: one corresponding to learning about the benefits of adoption (the invisible part) and one corresponding to best replying to the benefit of adopting the alternative action (the visible action part). The goal of this paper is to analyze the dynamic behavior of such a coupled system and to understand the influence of the network. In particular, we will explain the various collective behaviors as endogenous effects governed by the network.

We follow Jackson and Yariv [2005, 2007] in working with a stylized model of a social network. Each agent has a number of neighbors whose inclination and behavior influence the agent's inclination and decision. The inclination is the tendency of an agent to adopt new behavior or innovation, and influences the binary decision of the agent: a choice between $A$ and $B$. Here, action $A$ is the status quo and the agent switches to action $B$ if it appears worthwhile of doing so. This of course depends on the costs and benefits of switching, where the payoff depends on how many of an agent's neighbors have already switched. Each individual is characterized by the number of her neighbors, namely her degree, and her costs where the latter are randomly and independently assigned before the game starts. Exact local network structure cannot be efficiently used for large networks because of the complexity of the problem, thus we use averaged information instead of exact information. This "mean field approach" is done by assuming that agents have only partial information about the degrees and costs of the population in terms of a probability distribution over degrees and costs, but don't know with whom they are connected. In other words, there are no fixed neighbor sets. At the beginning of each period, individuals randomly choose their neighbors from the 
rest of the population according to their degrees. Furthermore, individuals start with an initial inclination towards adopting $B$ and update their inclinations by learning from those having already switched in their neighborhoods.

There are four main insights of our model: First, we can explain a variety of behavior of the adoption rates by means of the underlying inclination dynamics. Second, average inclination behaves as an orchestrating tool to control adoption behavior. Third, the notion of a tipping point has to be generalized as the critical mass of adoption depends on initial inclinations. Fourth, we show how equilibria and dynamic behavior change as we change the underlying network and other parameters.

Our abstract model and findings can be taken as a metaphor for many applications. For example, in sociology, it provides a step towards understanding the volatility of rioting behavior and the impact of the social network architecture. In marketing, our results contribute to understanding why a new product becomes a success or failure. In financial markets, the results could advance the understanding of market sentiments. ${ }^{1}$

The structure of this paper is as follows. Section 2 describes the strategic choice of adoption as a best response dynamics when the inclinations are given and vary exogenously. Section 3 introduces social learning as an endogenous process of inclination formation and shows how to couple learning to the best response dynamics of Section 2 . It also discusses equilibrium existence and structure for a selection of networks. Other dynamic properties are illustrated by numerical simulation. Section 4 concludes the paper. For reasons of clarity and readability all analytical proofs are shifted to the appendix.

\section{Diffusion Dynamics on Networks}

\subsection{The Model}

We consider a society of individuals, each of them chooses an action between two alternatives $A$ and $B$. Assume $A$ is the default behavior (the status quo). The possibility arises to switch to a new action $B$, for example, to adopt a new technology, or to learn another language. The set of actions is denoted as $\mathcal{A}=\{A, B\}$. The individuals form a network characterized by the degree distribution $P(d)$ for $d=1, \ldots, D$ where $\sum_{d=0}^{D} P(d)=1$, with the possibility that $D=\infty$. Agent $i$ 's degree is denoted by $d_{i}$. We assume that each individual only knows how many neighbors she has, but does not know who those neighbors are. Such a situation may arise when the decision to adopt is based on previous observations. In that case, each individual has a certain estimation of the number of

\footnotetext{
${ }^{1}$ Here, we think of "sentiment" of an investor as the attitude of investors as to anticipated price developments in a market, and of 'market sentiment' as the general prevailing attitude.
} 
people she will interact with in the future. ${ }^{2}$ Put

$$
\widetilde{P}(d)=\frac{P(d) d}{\bar{d}}, \quad \bar{d}=\sum_{k=0}^{D} P(k) k .
$$

Here, $\widetilde{P}(d)$ represents the probability that a random chosen neighbor of a random individual in the network has degree $d$ whereas $\bar{d}$ denotes the average degree of the network. ${ }^{3}$ It is easy to see that $\sum_{d=1}^{D} \widetilde{P}(d)=1$, which means that $\widetilde{P}(d)$ is a proper probability distribution.

Agent $i$ 's utility from adopting action $a$ is given by $u\left(a, d_{i}, x_{i}\right)$, where $d_{i}$ is her degree and $x_{i}$ is her estimation of the fraction of individuals switching to $B$. Let $v\left(d_{i}, x_{i}\right)$ be defined as

$$
v\left(d_{i}, x_{i}\right)=u\left(B, d_{i}, x_{i}\right)-u\left(A, d_{i}, x_{i}\right),
$$

representing the benefit from switching from $A$ to $B$. Each agent $i$ has an idiosyncratic $\operatorname{cost} c_{i}>0$ of switching which we will specify shortly. Agent $i$ switches to behavior $B$ if her benefit is no less than her cost, i.e.,

$$
v\left(d_{i}, x_{i}\right) \geq c_{i}
$$

Let $v(d, x)=g(d) x$ where $g(d)$ is a function capturing how the number of neighbors affects the individual benefit of adopting $B$. For example, if $g^{\prime}(d)>0$ then agents with higher degrees are more likely to adopt than those with less neighbors. With this functional form the adoption rule (3) reads

$$
g\left(d_{i}\right) x_{i} \geq c_{i}
$$

We assume the costs $c_{i}$ are randomly and independently assigned across the population according to a probability distribution. We follow Jackson and Yariv [2005] and discuss effects of benefit cost ratio $g(d) / c$. This implies that for each agent we consider the random variable $1 / c_{i}$ instead of $c_{i}$. Let $F: \mathbb{R}_{+} \rightarrow[0,1]$ be the cumulative distribution function of $1 / c_{i}$. Rearranging (4) provides

$$
\frac{1}{c_{i}} \geq \frac{1}{g\left(d_{i}\right) x_{i}}
$$

Individual $i$ 's probability of choosing action $B$ is therefore $1-F\left(1 /\left[g\left(d_{i}\right) x_{i}\right]\right)$. For mathematical simplicity, we assume that $F$ is continuous and twice differentiable.

\footnotetext{
${ }^{2}$ Methodologically, the approach is mean-field approximation.

${ }^{3}$ For a derivation of (1) see [Newman, 2010, Chapter 13.3].
} 
Remark 1. (i) Each individual agent $i$ knows only her own degree $d_{i}$ and her cost $c_{i}$ which are independently assigned before the game starts. Agents have only partial information about the degrees and costs of the population, given by $P$ and $F$. Thus, the model corresponds to a Bayesian game in the Harsanyi sense where the types of the game are given by vector $\left(d_{i}, c_{i}\right)$.

(ii) Inequality (4) can be rewritten as

$$
x_{i} \geq \frac{c_{i}}{g\left(d_{i}\right)}
$$

where the fraction $c_{i} / g\left(d_{i}\right)$ on the right hand side can be thought as the threshold of individual $i$ for adopting action B. In the classic model of Granovetter [1978], the "threshold" is "the number or proportion of others who must make one decision before a given actor does so" (see also Schelling [1971]).

\subsection{Dynamics and Equilibria}

At $t=0$, a fraction of $x^{0}$ of the population is exogenously switched to adopt action $B$. At each time $t>0$ ( $t$ is discrete), each individual, including those individuals whose initial adoption is $B$, updates her estimated (or believed) adoption rate based on the actual adoption rate in period $t-1$, and makes her decision of adoption. Denote the adoption rate in period $t$ by $x^{t}$.

Here we assume that the individuals are myopic, best respond to the state of the last period. Let $x_{d}^{t}$ denote the fraction of individuals who have degree $d$ and adopt action $B$ in period $t$. Then, for each $t>0$,

$$
x_{d}^{t}=1-F\left(\frac{1}{g(d) x^{t-1}}\right),
$$

where $x^{t}$ represents the link-weighted average of $x_{d}^{t}$, i.e.,

$$
x^{t}=\sum_{d} \widetilde{P}(d) x_{d}^{t}
$$

Equations (7) and (8) can be interpreted as follows. All individuals with the same degree are indistinguishable, therefore they have the same probability of adopting action $B$, as described in Equation (7). Since individuals cannot specify their neighbors, the estimation of $x^{t}$ is based on expectation. A random neighbor with degree $d$ has probability $\widetilde{P}(d)$ to be chosen, and the chosen neighbor has in turn a probability of $x_{d}^{t}$ to adopt action $B$ at time $t$, hence the expected adoption rate $x^{t}$ is the weighted average of $x_{d}^{t}$ as shown in Equation (8).

Combining (7) and (8) provides

$$
x^{t}=h\left(x^{t-1}\right):=1-\sum_{d} \widetilde{P}(d) F\left(\frac{1}{g(d) x^{t-1}}\right),
$$


and the following simple equation characterizes equilibria

$$
x=h(x)=1-\sum_{d} \widetilde{P}(d) F\left(\frac{1}{g(d) x}\right) .
$$

\subsection{Diffusion Process}

Jackson and Yariv [2005] analyze the model for $g(d)=\alpha \cdot d^{\beta}$ where $\alpha$ and $\beta$ are constants. ${ }^{4}$ The parameter $\alpha$ can be seen as a key factor of individual thresholds. It amplifies or decreases the effect of individual degrees on the overall adoption rate. As described in Equation (6), a larger $\alpha$ implies a lower individual threshold, which implies a higher individual inclination to adopt action $B$.

In our generalized model we will drop the assumption that $\alpha$ is a static parameter and allow it to change over time. The idea is to introduce a process of social learning. People observe empirical evidence from prior adopters in their neighborhood which influences their inclinations towards adopting the innovation. This translates into the model as a dynamic variable $\alpha^{t}$ which is determined endogenously by a learning process. Our purpose is to study its evolution and in particular how its behavior affects the dynamics of adoption.

To get some feeling for the influence of inclination $\alpha^{t}$ on the adoption rate $x^{t}$ let us discuss some simple cases first. The explicit learning dynamics will be introduced in Section 3.

Example 1. Assume $g$ to be constant, i.e., $g(d)=\alpha$ for all $d$, such that the benefit from choosing action $B$ does not depend on individual degrees. Here, Equation (7) simplifies to

$$
x_{d}^{t}=1-F\left(\frac{1}{\alpha x^{t-1}}\right)=x^{t} .
$$

Assume the inverse cost distribution $F$ to be uniform on $[0, b], b>0$. By definition, the uniform distribution on $[0, b]$ has cumulative distribution function

$$
F(y)= \begin{cases}0 & \text { for } y<0 \\ y / b & \text { for } y \in[0, b] \\ 1 & \text { for } y>b .\end{cases}
$$

Function $h(x)$ describing the dynamics of process $\left\{x^{t}\right\}$ given in (9) then takes the following simple form

$$
h(x)=1-F\left(\frac{1}{\alpha x}\right)= \begin{cases}0 & \text { for } x<\frac{1}{b \alpha} \\ 1-\frac{1}{b \alpha x} & \text { for } x \geq \frac{1}{b \alpha}\end{cases}
$$

\footnotetext{
${ }^{4}$ As an extension, Jackson and Yariv [2007] treated $v(d, x)$ as a general function without explicitly considering its shape.
} 
The dynamics of $\left\{x^{t}\right\}$ depend on the number of intersections between $h(x)$ and the $45^{\circ}$ line as illustrated in Figure 1 for different levels of $\alpha$. If $h(x)$ intersects the identity line twice, the lower interception point is an unstable equilibrium, usually referred to as a "tipping point". If the dynamics start above that point, the adoption rate $x^{t}$ increases monotonically to the upper intersection point, which is a stable equilibrium. Below the tipping point, the dynamics of $x^{t}$ decrease monotonically to zero. Closed forms of the equilibria easily follow from the fixed point equation $h(x)=1-\frac{1}{b \alpha x}=x$.

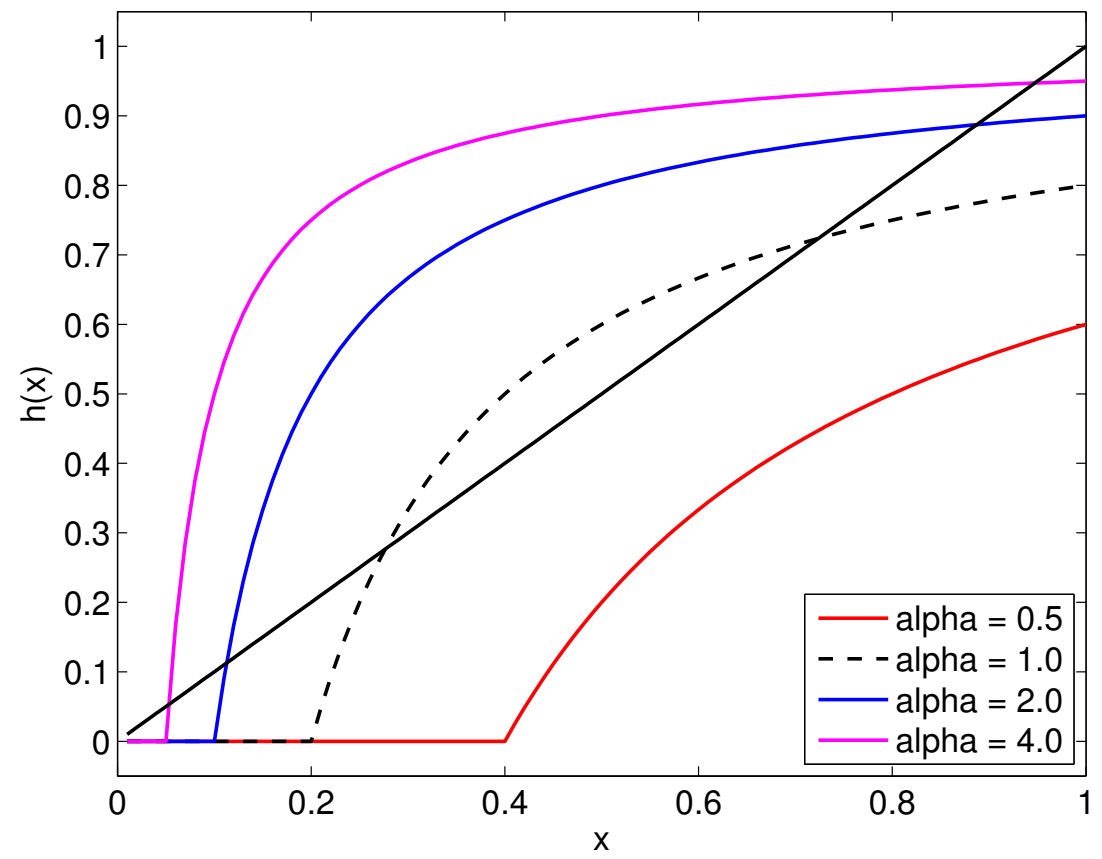

Figure 1: Dynamics of $x^{t}$ with $\alpha=0.5,1,2,4$ and $b=5$.

Now consider the effect of $\alpha$ changing over time, say, $\alpha^{t}$ is monotonically increasing. Interestingly, the effect on the adoption rate $x^{t}$ can be non-monotonic as illustrated by Figure 2. The dynamics start at low levels such that $x^{t}$ would to tend monotonically to zero for constant $\alpha$. However, since $\alpha^{t}$ "heats up" in the background, the graph of $h\left(x \mid \alpha^{t}\right)$ shifts to the top left quickly enough to "catch" the current level of $x^{t}$. In other words, individual thresholds decrease quickly enough such that the tipping point falls below $x^{t}$ in finite time.

Example 2 (continued from Example 1). Now instead of $F$ being uniform we assume that $F$ is a normal distribution with mean $\mu$ and variance $\sigma^{2}$ given by $F(y)=\Phi(y \mid \mu, \sigma)$. If $g$ is again constant, the dynamical process $x^{t}=h\left(x^{t-1}\right)$ of (9) is described by

$$
h(x)=1-\Phi\left(\frac{1}{\alpha x} \mid \mu, \sigma\right), \quad 0 \leq x \leq 1 .
$$

\footnotetext{
${ }^{5}$ See e.g. Jackson and Yariv [2007].
} 

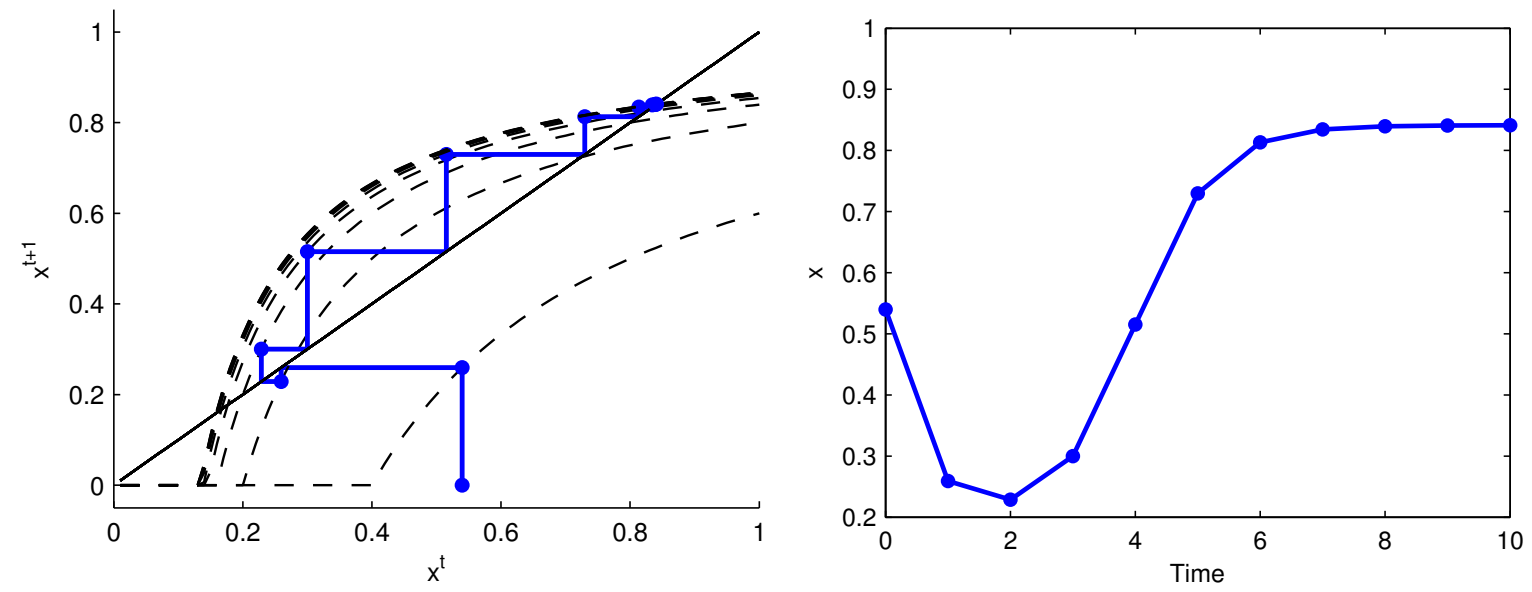

Figure 2: Dynamics of $\left\{x^{t}: t=0, \ldots, 10\right\}$ with $x^{0}=0.54$, where $\alpha^{t}=\alpha^{0}+\sum_{s=1}^{t}(1 / 2)^{s}$ for $t=1, \ldots, 10, \alpha^{0}=1 / 2$, and $b=5$. The dynamics follow $x^{t+1}=1-F\left(\frac{1}{\alpha^{t} x^{t}}\right)$.

This is the setting of the classical threshold model of Granovetter [1978]. Figure 3 illustrates the dynamics for different values of $\alpha$. Again, changing $\alpha^{t}$ can result in a variety

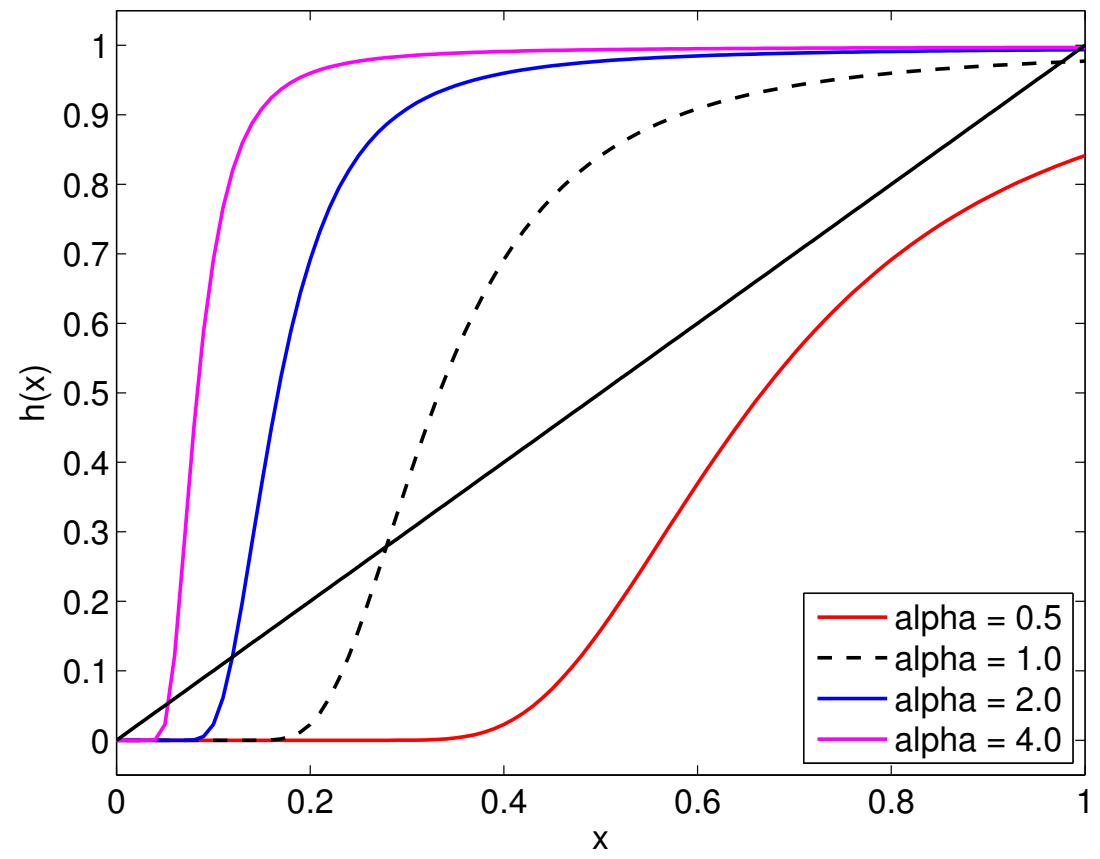

Figure 3: Dynamics of $x^{t}$ under $F \sim N(\mu=3, \sigma=1)$ with $\alpha=0.5,1,2,4$

of behavior of the adoption rate $x^{t}$.

The simplicity of Example 1 and 2 originates from the assumption of $g$ being constant in $d$ such that (9) simplifies considerably and the degree distribution $P(d)$ has no influence 
on the dynamical behavior. If $g$ is a function of degrees, however, the dynamics become more complicated in the sense that $h(x)$ is typically only piecewise differentiable. The reason is that the adoption rule in (5) generates different results for different degrees, such that individuals with different degrees start adopting earlier or later. The communication structure $P(d)$ averages over the different adoption rates, therefore leaving it's footprint on the dynamics of $x^{t}$.

Proposition 1. Suppose that $F$ is uniform on $[0, b]$ and $g$ given by $g(d)=\alpha d^{\beta}$ with constant $\alpha>0$ and $\beta>0$. Then $h(x)$ non-decreasing, continuous, and piecewise concave.

Proof Let $S_{d} \in[0,1]$ denote the level of overall adoption that starts triggering adoption of degree $d$ agents. From (7) it follows that $S_{d}$ is given by $\sup \left\{x \mid F\left[1 /\left(\alpha d^{\beta} x\right)\right]=0\right\}$. Since $\beta>0$ higher degrees have a higher inclination to adopt such that $S_{d^{\prime}}<S_{d}$ for all $d^{\prime}>d$. Hence for increasing $x$ the function $h(x)$ passes the points $S_{d}$ where a new class of agents with degree $d$ start joining, indicated by a jump in the curvature of $h(x)$.

In particular, the response dynamic of degree $d$ agents is given by

$$
h_{d}(x):=1-F\left(\frac{1}{\alpha d^{\beta} x}\right)= \begin{cases}0 & \text { for } x<S_{d} \\ 1-1 /\left(b \alpha d^{\beta} x\right) & \text { for } x \geq S_{d}\end{cases}
$$

which is strictly increasing, continuous and concave for $x \geq S_{d}$. From (10) it follows that

$$
\begin{aligned}
h(x) & =1-\sum_{d} \widetilde{P}(d) F\left(\frac{1}{\alpha d^{\beta} x}\right)=\sum_{d} \widetilde{P}(d)\left\{1-F\left(\frac{1}{\alpha d^{\beta} x}\right)\right\} \\
& =\sum_{d} \widetilde{P}(d) \times h_{d}(x),
\end{aligned}
$$

with

$$
h(x)=\sum_{k \geq d} \widetilde{P}(k) h_{k}(x) \quad \text { for } S_{d} \leq x<S_{d-1} .
$$

We conclude that $h(x)$ consists a sum of non-decreasing, continuous and concave functions and thus inherits these properties.

Remark 2. From Figure 1 in Jackson and Yariv [2005] it is tempting to jump to the conclusion that the effect of replacing $g=\alpha$ by $g(d)=\alpha d^{\beta}$ with $\beta>0$ is merely an upwards shift in adoption levels. However, the effect on the dynamics can be very complicated. For instance, multiple stable equilibria as well as tipping points may exist as shown in following example.

Example 3. Assume $g(d)=\alpha d^{\beta}$ with $\beta>0$ and suppose $F$ is uniform over $[0, b]$. Figure 4 gives an illustration for $\beta=2$. The dashed lines $h_{1}(x)$ and $h_{2}(x)$ show the adoption 
behavior for degree 1 and 2 individuals. The graph of $h_{2}(x)$ starts increasing at around 0.08, meaning that $8 \%$ overall adoption rate starts triggering the adoption of individuals with degree 2. Individuals with the lowest degree 1 are the last to join adoption with positive values of $h_{1}(x)$ starting at around 0.34 . The total number of adopters is the sum of the numbers of adopters with different degrees. As shown in Figure 4, function $h(x)$ can have multiple stable and unstable equilibria.

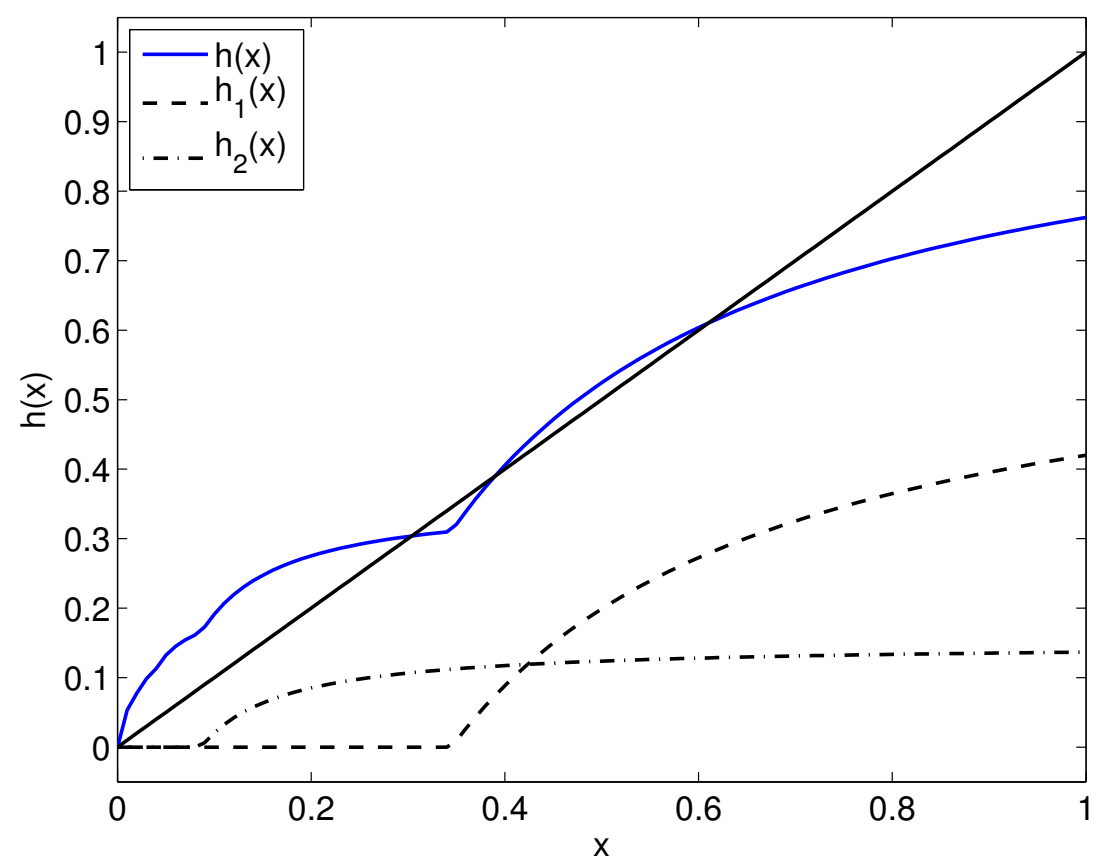

Figure 4: Dynamics of $x^{t}$ with $\beta=2, \alpha=1, P(d) \propto d^{-3.1}$ and $b=2.9$.

We now discuss the speed of convergence, especially for the situation that the initial adoption rate $x^{0}$ is between a tipping point and the next higher stable steady state. For constant $\alpha$ and $\beta=0$ Jackson and Yariv [2007] showed that the adoption rate over time exhibits an S-shape (see their Proposition 7). In other words, the speed of convergence accelerates first and then decelerates after the adoption rate passing a critical value. This property is not necessarily preserved under the model with time varying $\alpha^{t}$. The next example illustrates some effects on the speed of convergence.

Example 4. Suppose $\beta=0$ and let $\alpha^{t}$ take values $1,1,1.05,1.1,1.15,1.2,1.25,1.3$, 2.0, 6.0, 12.0 for $t=0, \ldots, 10$ respectively. Figure 5 illustrates the evolution of $x^{t}$. Up to $t=8$ the increasing speed of $\alpha^{t}$ is constant and $x^{t}$ indeed changes from accelerating to decelerating. For $t>8$, however, the rapid change of $\alpha^{t}$ results in a vigorous shift of $h(x)$ to the upper-left which leads again to an acceleration of $x^{t}$. In conclusion, the speed of convergence of $x^{t}$ depends on the dynamics of $\alpha^{t}$. 

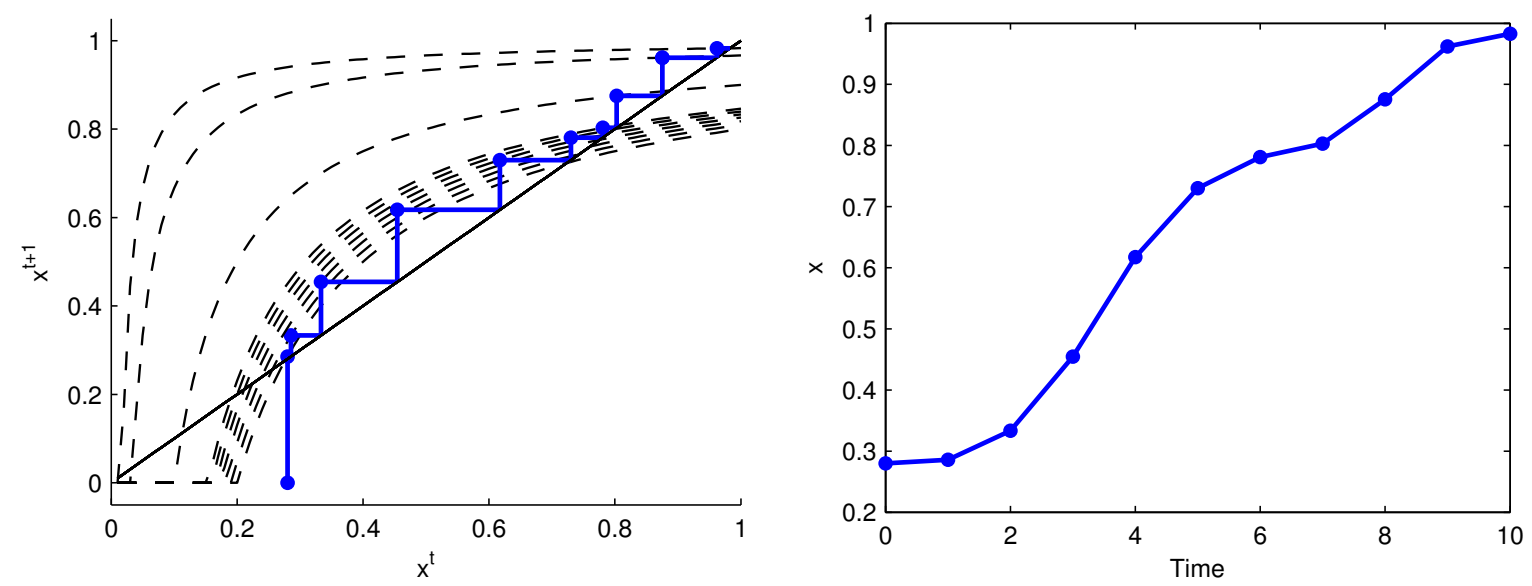

Figure 5: Dynamics of $\left\{x^{t}: t=0, \ldots, 10\right\}$ with $x^{0}=0.28$, where $\alpha^{t}=1,1,1.05,1.1$, $1.15,1.2,1.25,1.3,2.0,6.0,12.0$ for $t=0, \ldots, 10$, and $b=5$.

\section{Social Learning}

\subsection{The Model}

In this section we consider a class of social learning models in which agents rationally evaluate the observed evidence of prior adopters. The idea is that when an innovation becomes available - like e.g. a new technology or a new management strategy - people want to see how it works for others before trying themselves. As Young [2009] points out the literature on social learning is extensive. By means of some simplifying assumptions, however, he introduces a fairly general class of social learning models that still allows for heterogeneous characteristics. We will reintroduce his general framework by adding the feature that learning is governed by the social network. ${ }^{6}$ The next step will be to couple the learning mechanism to the diffusion model discussed in Section 2.

Consider again the function $g(d)=\alpha d^{\beta}$ which captures how the number of neighbors affects the individual benefits from adopting $B .{ }^{7}$ For each agent of degree $d$ let $\alpha_{d}^{0} \in(0, \infty)$ denote the initial inclination towards switching behavior. As time proceeds information from adopters keeps coming in and agents update their inclinations. Let the average

\footnotetext{
${ }^{6}$ Young [2009] models flow of information by a Poisson arrival process including a parameter $\beta_{i}$ measuring the extent to which agent $i$ "gets around" which could be interpreted as a degree in a social network. He notes, however, that this parameter will not be sufficient to describe impact of the network topology on the dynamics. (See his footnote 16.)

${ }^{7}$ Young [2009] assumes that $g(d)=A d^{\beta}$, where $A$ is a normally distributed random variable with mean $\mu>0$ and variance $\sigma^{2}$, independent and identically distributed among agents and time periods. Here, $\mu d^{\beta}$ is the mean individual payoff gain per period of switching to $B$. Ex ante, however, agents are not informed about the true value of $\mu$ and start with different inclinations at $t=0$. In order to demonstrate dynamic effects of initial expectations we leave the initial levels as a degree of freedom.
} 
inclination at time $t \geq 0$ be given by

$$
\alpha^{t}=\sum_{d} \widetilde{P}(d) \alpha_{d}^{t}
$$

For $t>0$, each individual will have met a random draw of neighbors at $t-1$. If an individual has $d$ neighbors and the fraction of adopters is $x^{t-1}$, the number of independent observations of adopters is $d x^{t-1}$, and her updated inclination $\alpha_{d}^{t}$ is defined as the weighted average of her own initial inclination $\alpha_{d}^{0}$ and the old average inclination $\alpha^{t-1}$, i.e.,

$$
\alpha_{d}^{t}=\frac{\tau_{d} \alpha_{d}^{0}+d x^{t-1} \alpha^{t-1}}{\tau_{d}+d x^{t-1}}
$$

where $\tau_{d} \in(0, \infty)$ reflects flexibility in learning (see Groot [1970], Young [2009]). Low values of $\tau_{d}$ indicate that relatively little evidence is necessary to change agent $i$ 's inclination. ${ }^{8}$ The term $d x^{t-1}$ is equivalent to the number of adopting neighbors. Note that there is no updating if this term is zero, i.e. there is no learning in absence of evidence.

It is now an easy task to combine the learning dynamics (16) with the dynamics of adoption as discussed in the previous section. Using Equations (9), (15) and (16), the dynamical process of average inclination $\alpha^{t}$ and adoption rate $x^{t}$ is given by

$$
\begin{aligned}
\alpha^{t} & =\sum_{d} \widetilde{P}(d) \frac{\tau_{d} \alpha_{d}^{0}+d x^{t-1} \alpha^{t-1}}{\tau_{d}+d x^{t-1}}, \\
x^{t} & =1-\sum_{d} \widetilde{P}(d) F\left(\frac{1}{d^{\beta} \alpha^{t-1} x^{t-1}}\right) .
\end{aligned}
$$

What remains to be checked are the initial conditions. For notational convenience we put $\left\{\alpha_{d}^{0}\right\}:=\left\{\alpha_{d}^{0}\right\}_{d=1}^{D}$. Note that by explicit inclusion of initial inclinations in (17) implies that two societies starting at equal average levels $\left(\alpha^{0}, x^{0}\right)$ can display different dynamical behavior if the average $\alpha^{0}$ stems from a different profile of individual inclinations $\left\{\alpha_{d}^{0}\right\}$.

Let $H: \mathbb{R}_{+} \times[0,1] \rightarrow \mathbb{R}_{+} \times[0,1]$ denote the the right hand side of (17) and (18), i.e.,

$$
\left[\begin{array}{l}
\alpha^{t} \\
x^{t}
\end{array}\right]=H\left(\begin{array}{l}
\alpha^{t-1} \\
x^{t-1}
\end{array} \mid\left\{\alpha_{d}^{0}\right\}\right), \quad H\left(\begin{array}{l}
\alpha \\
x
\end{array} \mid\left\{\alpha_{d}^{0}\right\}\right)=\left[\begin{array}{c}
\sum_{d} \widetilde{P}(d) \frac{\tau_{d}^{0} \alpha_{d}^{0}+d x \alpha}{\tau_{d}^{0}+d x} \\
1-\sum_{d} \widetilde{P}(d) F\left(\frac{1}{d^{\beta} \alpha x}\right)
\end{array}\right] .
$$

The initial inclinations enter (17) explicitly has subtle effects on notions of equilibria. We should carefully distinguish between equilibrium behavior on the aggregate dynamics (17) and the dynamics of individual inclinations (16). Consider a state $\left(\left\{\alpha_{d}\right\}, x\right)$ with the property that individual inclinations and adoption rate don't change when chosen as starting point. It easily follows from (16) that for $x>0$ this is the case if and only

\footnotetext{
${ }^{8}$ This definition corresponds to an infinite memory property, which means that every individual remembers her initial inclination and updates it forever. There are other possible definitions, e.g., replacing $\tau_{d} \alpha_{d}^{0}$ by $\tau_{d} \alpha_{d}^{t-1}$.
} 
if $\alpha^{0}=\alpha_{d}^{0}$. The intuition is straightforward. If inclinations are identical there is no learning. We shall refer to this scenario simply as symmetric equilibrium.

Now assume we observe that average inclination and adoption rate haven't changed from the beginning. What conclusions can we draw about the individual inclinations? One possibility is that the individual inclinations are also constant. We concluded that this can only be the case when the individual inclinations are equal. Another possibility is that the individual inclinations change while keeping the average $\alpha$ constant. For example, a possible scenario from $t=0$ to $t=1$ is that initial inclinations $\left\{\alpha_{d}^{0}\right\}$ changed to $\left\{\alpha_{d}^{1}\right\}$ while $\alpha^{1}=\alpha^{0}$. Can this happen more than once? The answer is no because from (16) it follows that if average inclination $\alpha^{t}$ and adoption rate $x^{t}$ stay constant so does $\alpha_{d}^{t}$. We summarize our observations.

Proposition 2. Assume the aggregate dynamics (17) stays in an equilibrium $\left(\alpha^{*}, x^{*}\right)$ with $x^{*} \neq 0$ when starting in a state $\left(\left\{\alpha_{d}^{0}\right\}, x^{*}\right)$. Then the individual inclinations following (16) are either $(i)$ constant and equal, or (ii) update once to a level $\left\{\alpha_{d}^{1}\right\} \neq\left\{\alpha_{d}^{0}\right\}$ and stay there.

This one time adjustment shall be represented by $\left(\left\{\alpha_{d}^{0}\right\},\left\{\alpha_{d}^{1}\right\}, x\right)$ and we will refer to it simply as average equilibrium.

Finally, there is the more general case that the trajectory of $\left(\left\{\alpha_{d}^{t}\right\}, x^{t}\right)$ converges to a limit $\left(\left\{\alpha_{d}^{*}\right\}, x^{*}\right)$. We shall refer to the corresponding average levels $\left(\alpha^{*}, x^{*}\right)$ as an attractor. We summarize the definitions.

Definition 1. An equilibrium of (19) is represented by the triple $\left(\left\{\alpha_{d}^{0}\right\},\left\{\alpha_{d}^{1}\right\}, x\right)$ such that

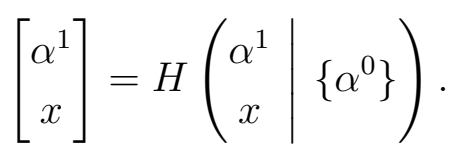

where $\alpha^{1}=\sum_{d} \widetilde{P}(d) \alpha_{d}^{1}=\alpha^{0}$. A pair $\left(\alpha^{*}, x^{*}\right) \in \mathbb{R}_{+} \times[0,1]$ is an attractor of (19) if there exists a sequence $\left(\left\{\alpha_{d}^{t}\right\}, x^{t}\right)$ such that

$$
\lim _{t \rightarrow \infty}\left(\alpha^{t}, x^{t}\right)=\left(\alpha^{*}, x^{*}\right)
$$

Remark 3. If we let $\tau_{d}=0$ in (16), individual inclinations become memoryless which are simply the average inclination of the previous period, i.e.,

$$
\alpha_{d}^{t}=\alpha^{t}=\sum_{d} \widetilde{P}(d) \alpha_{d}^{t-1}
$$

This leads to an immediate agreement of individual inclinations to $\alpha^{0}$ in the very first period after the process started. On the contrary, if we let $\tau_{d} \rightarrow \infty$, every individual will 
keep her initial inclination forever and not update at all. Interestingly, the average inclination will be $\alpha^{t}=\alpha^{0}$ for $t>0$, which coincides with the case of $\tau_{d}=0$. Consequently, in both cases, the dynamical process is reduced to the one-dimensional system discussed in Section 2.1.

The complexity of the dynamical system makes it almost impossible to find closed form solutions for general settings. In the upcoming sections we will discuss properties of (19) for some special cases of networks and costs. The following section discusses networks with a small number of possible degrees.

\subsection{Equilibrium existence and structure}

The question on existence of equilibria is straightforward to answer. In absence of adoption, $x=0$, there is no learning from adopters and hence no inclinations are ever updated as indicated by (16) and (17). With respect to adoption note that the right hand side of (18) is zero for $x=0$. Hence the best response to a zero adoption level is again zero. We shall refer to equilibria with zero adoption rate as trivial equilibria. The question is now whether there are any non-trivial equilibria of system (19). Here, we provide an analytical discussion for networks with low dimensions, i.e., a small number of possible degrees.

Proposition 3. Assume the support of the network $P$ is given by $\left\{d_{1}, d_{2}\right\}$. Then the existence of non-trivial equilibria is guaranteed by either (1) the flexibility ratio $\tau_{d_{1}} / \tau_{d_{2}}$ is equal to the degree ratio $d_{1} / d_{2}$, or (2) the system starts with identical initial inclinations.

Proposition 3 implies that under the condition of $\tau_{d_{1}} / \tau_{d_{2}} \neq d_{1} / d_{2}$, any non-trivial equilibrium is symmetric.

Proposition 4. Assume the support of the network $P$ is given by $\left\{d_{1}, d_{2}, d_{3}\right\}$ and $F \sim$ $\mathrm{U}[0, b]$. Put $\tau_{d}=\tau>0$ and $\beta=0$. Without loss of generality, we also assume $d_{1}<d_{2}<$ $d_{3}$. It is possible to have non-trivial equilibria if either $\alpha_{d_{1}}^{0}=\alpha_{d_{3}}^{0} \neq \alpha_{d_{2}}^{0}$, or $\alpha_{d_{i}}^{0}$ 's are all different and not monotonic in $d$.

Example 5. Assume a network with degrees 1,2 and 3 given by $\widetilde{P}(1)=\frac{1}{4}, \widetilde{P}(2)=\frac{1}{2}$, $\widetilde{P}(3)=\frac{1}{4}$. Put $b=6$, and $\tau=0.5$. Inserting

$$
\left(\alpha_{1}^{0}, \alpha_{2}^{0}, \alpha_{3}^{0}\right)=\left(\frac{1}{2}, 1, \frac{1+x}{2+6 x}\right)
$$

into (16) provides

$$
\left(\alpha_{1}^{1}, \alpha_{2}^{1}, \alpha_{3}^{1}\right)=\left(\frac{4 x+1}{6 x+2}, \frac{2 x+1}{3 x+1}, \frac{4 x+1}{6 x+2}\right)
$$


while $\alpha^{0}=\alpha^{1}=(8 x+3) /(12 x+4)=\alpha^{*}$. Inserting $\alpha^{*}$ into (18) and solving the fixed point equation provides $x^{*} \approx 0.3828$ or $x^{*} \approx 0.6032$.

Analogously, inserting

$$
\left(\alpha_{1}^{0}, \alpha_{2}^{0}, \alpha_{3}^{0}\right)=\left(1, \frac{1}{2}, \frac{1+4 x}{1+3 x}\right)
$$

into (16) provides

$$
\left(\alpha_{1}^{1}, \alpha_{2}^{1}, \alpha_{3}^{1}\right)=\left(\frac{5 x+2}{6 x+2}, \frac{5 x+1}{6 x+2}, \frac{5 x+2}{6 x+2}\right)
$$

while $\alpha^{0}=\alpha^{1}=(10 x+3) /(12 x+4)=\alpha^{*}$. Inserting $\alpha^{*}$ into (18) and solving the fixed point equation provides $x^{*} \approx 0.3027$ or $x^{*} \approx 0.7083$.

For more general cases, limit behavior and existence of attractors can be shown by simulation. Here we choose several initial conditions and track the paths over a long period. In Table 1 the convergence can be observed for all the three initial values, where one of them converges to a trivial attractor $\left(\alpha^{0}, 0\right)$, and two others converge to non-trivial attractors. The initial inclinations $\left\{\alpha_{d}^{0}\right\}$ are taken as a decreasing sequence in $d$.

Table 1: Convergence of the system

$$
\begin{aligned}
& \begin{array}{cccc} 
& \left(\alpha^{t}, x^{t}\right) & \left(\alpha^{t}, x^{t}\right) & \left(\alpha^{t}, x^{t}\right) \\
\hline t=0 & (0.4800,0.4900) & (0.3400,0.8900) & (0.3800,0.8900)
\end{array} \\
& t=10 \quad(0.5332,0.7470) \quad(0.3400,0.0000) \quad(0.4339,0.6223) \\
& t=20 \quad(0.5456,0.7576) \quad(0.3400,0.0000) \quad(0.4460,0.6582) \\
& t=50 \quad(0.5502,0.7612) \quad(0.3400,0.0000) \quad(0.4496,0.6661) \\
& t=100 \quad(0.5503,0.7613) \quad(0.3400,0.0000) \quad(0.4497,0.6662) \\
& t=200 \quad(0.5503,0.7613) \quad(0.3400,0.0000) \quad(0.4497,0.6662) \\
& \tau_{d}=\tau=0.2, \beta=0, F \sim U(0,10), d \in\{1, \ldots, 1000\}, \text { and } P(d) \propto d^{-2.5} \text {. }
\end{aligned}
$$

\subsection{Long run behavior and convergence: simulation}

The present section is intended to illustrate the dynamics of (19). In particular, we will discuss the case $\beta=0$ where an agent's utility depends on the fraction of neighbors who have adopted the action $B$. For the costs we assume the benchmark case $F \sim U[0, b]$. In 
this special setting (18) simplifies to

$$
\begin{aligned}
x^{t+1} & =1-\sum_{d} \min \left\{1, \frac{1}{b \alpha^{t} x^{t}}\right\} \widetilde{P}(d)=\sum_{d} \widetilde{P}(d)\left[1-\min \left\{1, \frac{1}{b \alpha^{t} x^{t}}\right\}\right] \\
& = \begin{cases}0 & \text { for } x^{t}<1 /\left(b \alpha^{t}\right) \\
1-1 /\left(b \alpha^{t} x^{t}\right) & \text { for } x^{t} \geq 1 /\left(b \alpha^{t}\right) .\end{cases}
\end{aligned}
$$

Hence the interaction network $P$ orchestrates entirely via updating inclinations (17). We will first prove a classification of local behavior in the phase plane. The first insight is that the domain $\mathbb{R}_{+} \times[0,1]$ of average values $\left(\alpha^{t}, x^{t}\right)$ can be divided into two areas by a function of $x$, where $\left(\alpha^{t}, x^{t}\right)$ exhibits different behaviors in different areas.

Proposition 5. Consider a process $\left(\alpha^{t}, x^{t}\right)$. There exists a function

$$
f(x)=\frac{1}{b(1-x) x}
$$

such that $x^{t+1}>x^{t}$ if $\alpha^{t}>f\left(x^{t}\right), x^{t+1}=x^{t}$ if $\alpha^{t}=f\left(x^{t}\right)$, and $x^{t+1}<x^{t}$ otherwise.

Proposition 5 can be explained as follows. For the points above (below) the curve $\alpha=f(x), x^{t}$ increases (decreases) in time. On the curve $\alpha=f(x)$ the adoption rate $x^{t}$ freezes for one period after which it follows the increasing/decreasing behavior of $\alpha^{t}$. We conclude that Proposition 5 rules out cyclic behavior.

Remark 4. Function (20) generalizes the notion of a 'tipping point' (see e.g. Jackson and Yariv [2007]). For constant $\alpha$ the solutions to $f(x)=\alpha$ are the lower unstable equilibrium (tipping point) and the higher stable equilibrium.

For the simulation we choose a scale free network $P(d) \propto d^{-2.5}$ with a maximum degree $D=1000, \tau=0.2$ and $b=10$. The dynamics are qualitatively similar to different values of $\tau$ and $b$ larger than $0,{ }^{9}$ however, it turns out that the initial levels of individual inclinations have a subtle impact. In the first run we will choose them such that $\alpha^{1}>\alpha^{0}$ and compare it with the opposite case afterwards. ${ }^{10}$

Figure 6 depicts the vector field of $\left(\alpha^{t}, x^{t}\right)$ for $t=0,1,2,3,5$ and 10 for different values of $\left(\alpha^{0}, x^{0}\right)$. In each dot the line indicates the moving direction. ${ }^{11}$ The color of

\footnotetext{
${ }^{9}$ In a nutshell, higher values of $\tau$ lead to faster convergence. The level of $b$ influences the shape of the attractor as shown in Proposition 5.

${ }^{10}$ This can easily be satisfied by a decreasing series of $\left\{\alpha_{d}^{0}\right\}$ in $d$. In order to have a somewhat systematic approach we choose a set of values $\left\{x_{m}^{0} \mid 0 \leq m \leq 100\right\}$ with $x_{i}^{0}=0.01 m$, and a set of vectors $\left\{\left\{\alpha_{n, d}^{0}\right\} \mid 0 \leq n \leq 100\right\}$ where $\alpha_{n, d}^{0}=\sum_{d=1}^{D} \widetilde{P}(d) \sum_{k=1}^{d} P(k)-\sum_{k=1}^{d} P(k)+0.02 n$. For each $n$, It follows that $\alpha_{n}^{0}=\sum_{d} \widetilde{P}(d) \alpha_{n, d}^{0}=0.02 n \geq 0$. Furthermore, $\left\{\alpha_{n, d}^{0}\right\}$ is decreasing in $d$, and as a result, $\alpha_{n}^{1}>\alpha_{n}^{0}$.

${ }^{11}$ The magnitude of speed is not shown in these plots as the variation of speed with different positions can be quite large.
} 
dots gives a classification of moving directions, e.g., red corresponds to increase in both axes while blue corresponds to increase in inclinations but decrease in adoption rate. Other directions are also represented by distinguished colors. The gray curve illustrates $\alpha=f(x)$ given by (20). Any trajectory starting above $\alpha=f(x)$ moves to the upper right and eventually converge to some point on the curve. The behavior below $\alpha=f(x)$ depends on the location. Trajectories starting with sufficiently high $\alpha^{0}$ cross the curve $\alpha=f(x)$ and merge with the behavior of those starting above $\alpha=f(x)$ to begin with. The crucial value of $\alpha^{0}$ to exhibit this type of behavior depends on initial adoption rate. Starting with an initial average inclination lower than the crucial value, however, leads the trajectory to end up in a trivial attractor. The crucial $\alpha^{0}$ will be further depicted in Figure 9 and 10.

Three typical time series of $\left(\alpha^{t}, x^{t}\right)$ with different initial values are depicted in Figure 7. The interesting behavior is the black path which starts only slightly different compared to the blue path. In the first few time periods the dynamic behavior is comparable, however, eventually the black path takes a turnoff, crosses and shows a similar evolution of red path. This implies that the trajectory of $x^{t}$ is not monotone but decreases first and turns to increasing later. Recall that this is the behavior we observed in Figure 2, where different levels of $\alpha$ were exogenously given.

We now turn to the case $\alpha^{1}<\alpha^{0}$ which will be described by choosing a linear increasing $\left\{\alpha_{d}^{0}\right\} .{ }^{12}$ Typical sample paths under the new initial condition are shown in Figure 8-(a). The major difference in comparison to Figure 7 are the trajectories starting above the grey curve at in the area $\alpha \geq f(x)$. Here, both the purple and the red path cross the curve $\alpha=f(x)$ from above at some point in time, and then continue with decreasing $x^{t}$. The red and black paths are eventually attracted to non-trivial attractors. The initial level $\alpha^{0}$ for the other trajectories, however, were too low to ensure adoption in the long run.

The distinguished areas of Figure 9 and 10 are called 'basin of attraction'. The red area includes initial values whose path will be attracted to a point on $\alpha=f(x)$, i.e., a non-trivial attractor, whereas all other points lead to a trivial attractor. For lower values of $x^{0}$ the border between the two areas merges with $\alpha=f(x)$.

As a conclusion of the current subsection, we summarize the long run behavior of our two-dimensional model as follows:

- There is a curve $\alpha=f(x)$ defined by Equation (20) which divides the domain $\mathbb{R}_{+} \times[0,1]$ into two areas in which $x^{t}$ behaves differently.

- Monotonic behavior of $\alpha^{t}$ can be observed in the initial phase of the process.

\footnotetext{
${ }^{12}$ Keeping other parameters unchanged, we let $\alpha_{n, d}^{0}=0.2+0.01 d+0.02 n$. It then follows that $\alpha^{1}<\alpha^{0}$ for all $n$.
} 

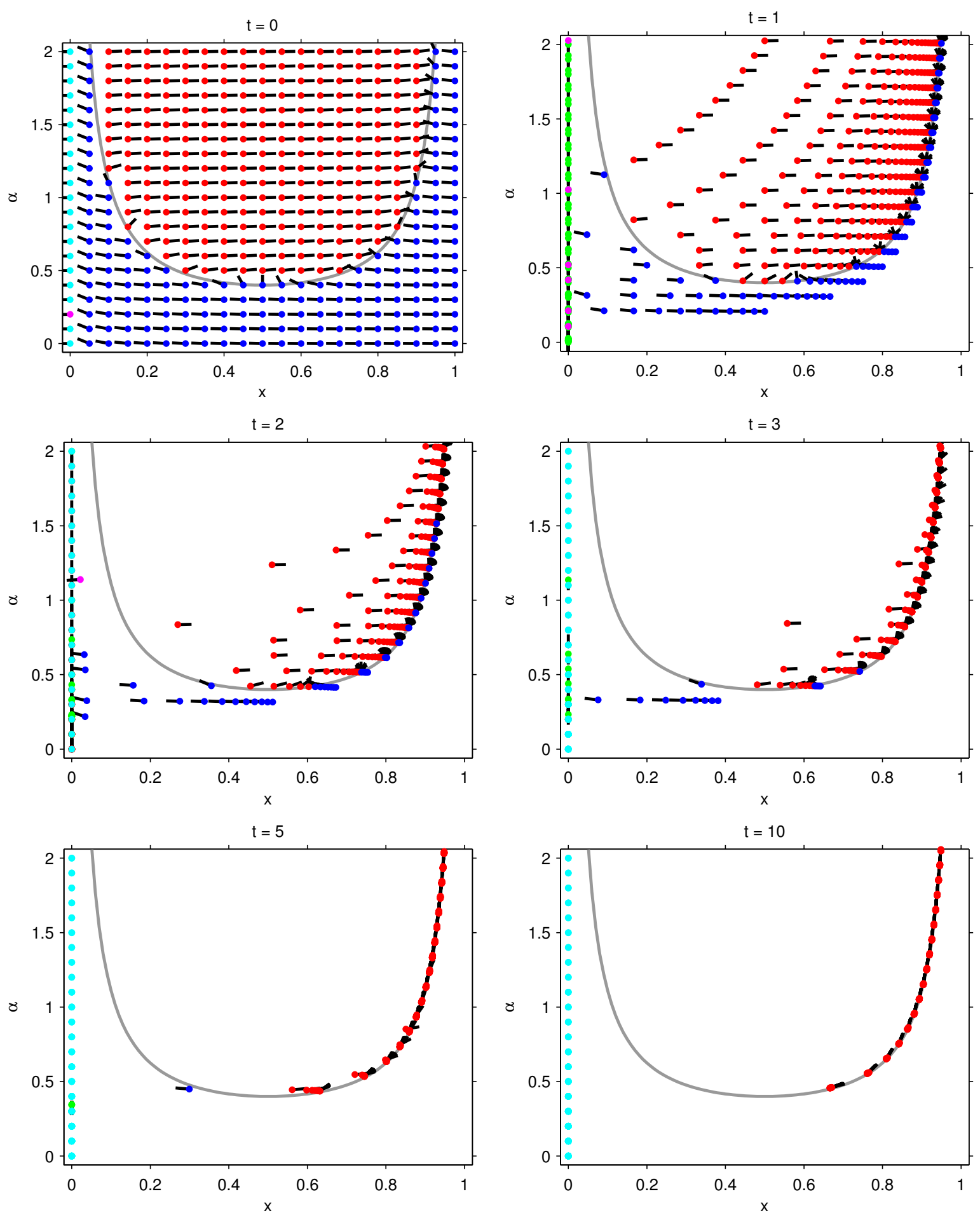

Figure 6: $2 \mathrm{D}$ vector plot of $\left(x_{m}^{t}, \alpha_{n}^{t}\right)$ for $t=0,1,2,3,5,10$ 


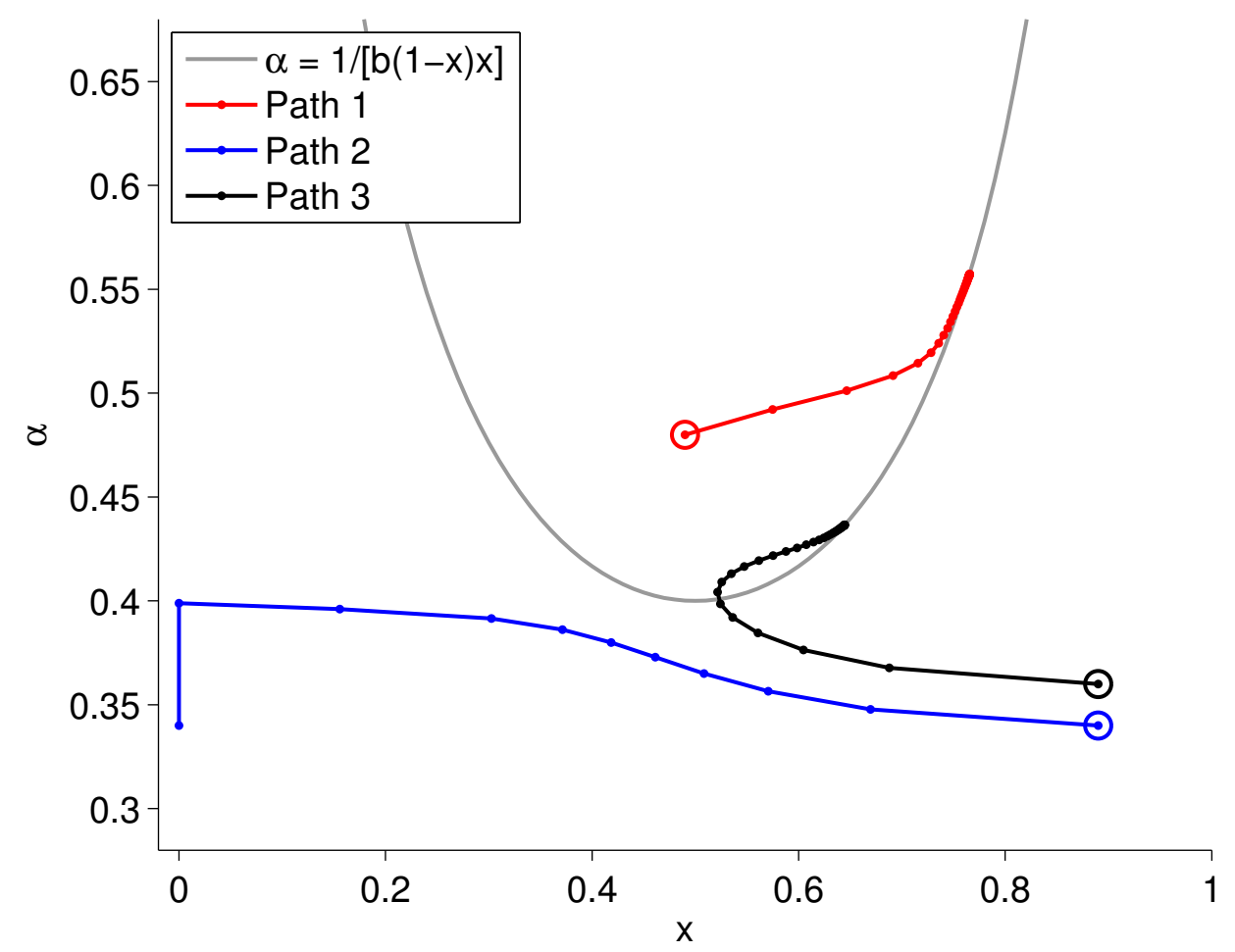

(a) Sample paths starting with different initial values

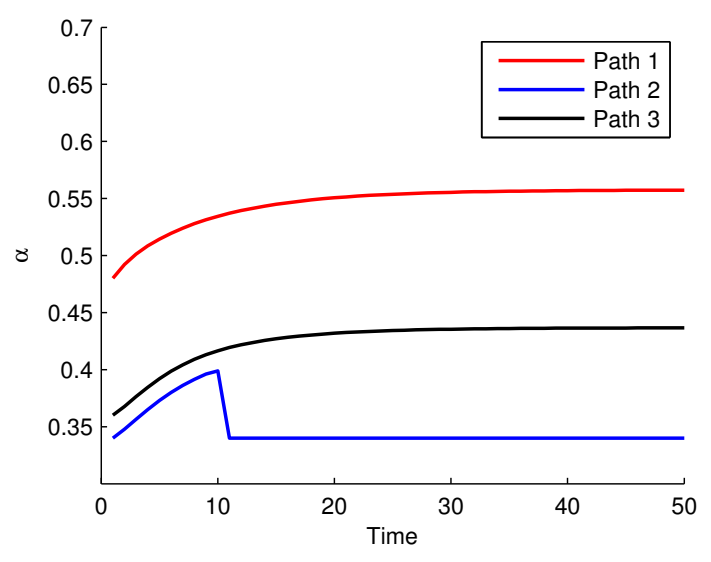

(b) Dynamical behavior of sentiment

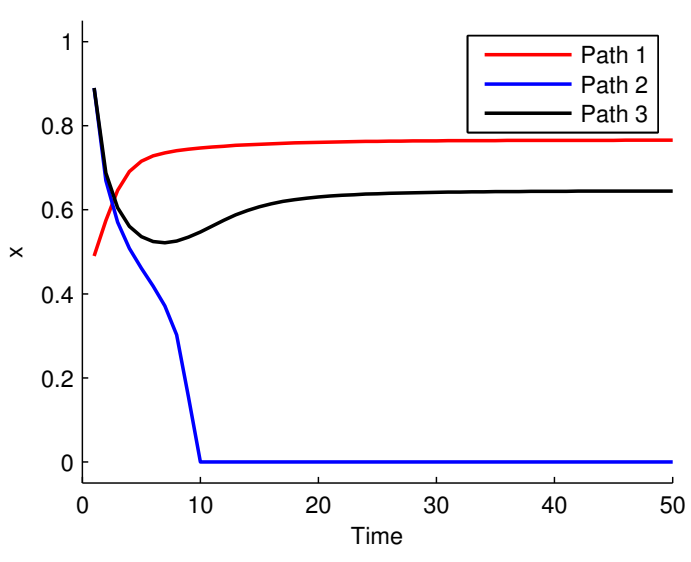

(c) Dynamical behavior of adoption rate

Figure 7: Three typical paths of $\left(\alpha^{t}, x^{t}\right)$ satisfying $\alpha^{1} \geq \alpha^{0}$. In subfigure (a) points with a circle indicate the initial values. 


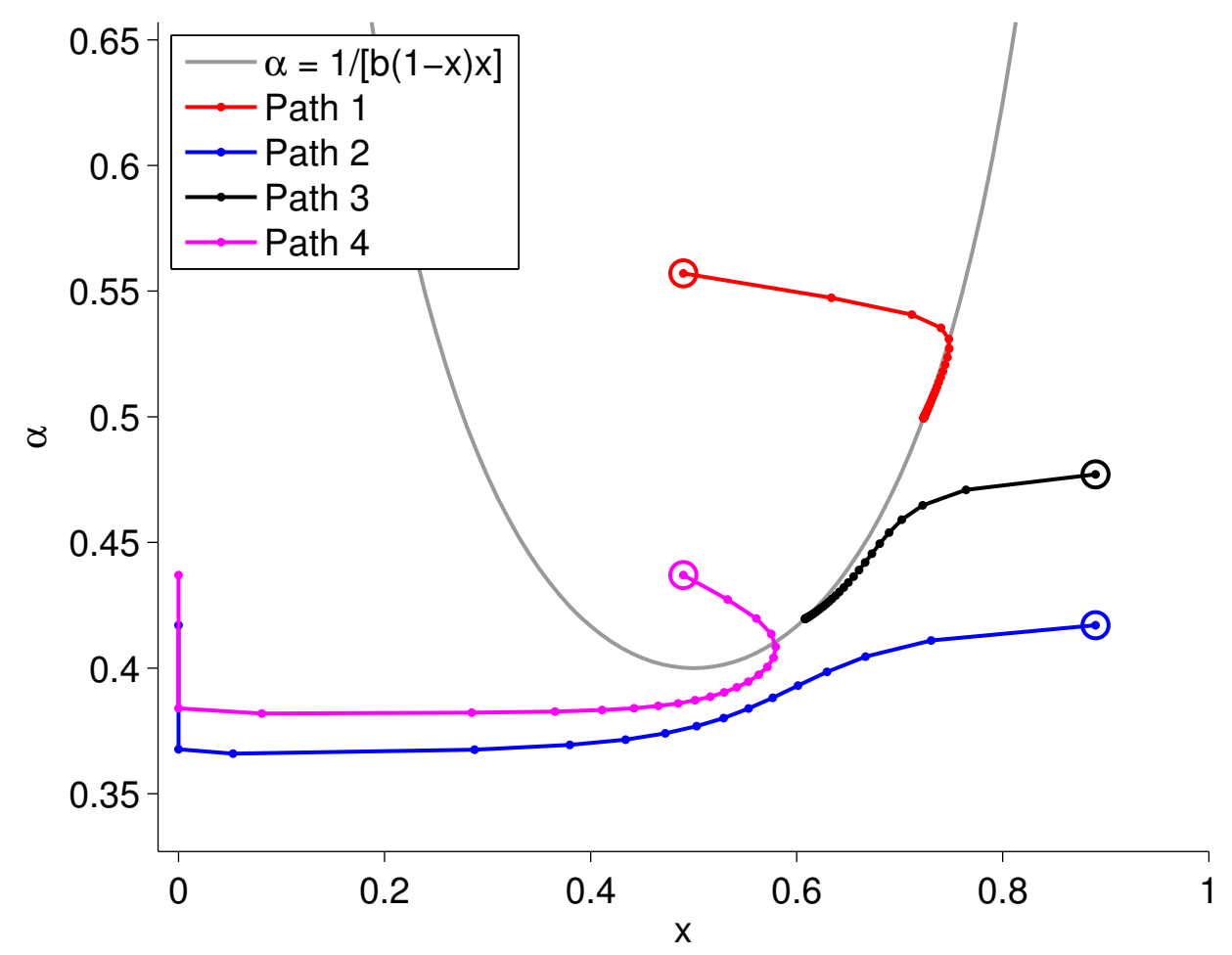

(a) Sample paths starting with different initial values

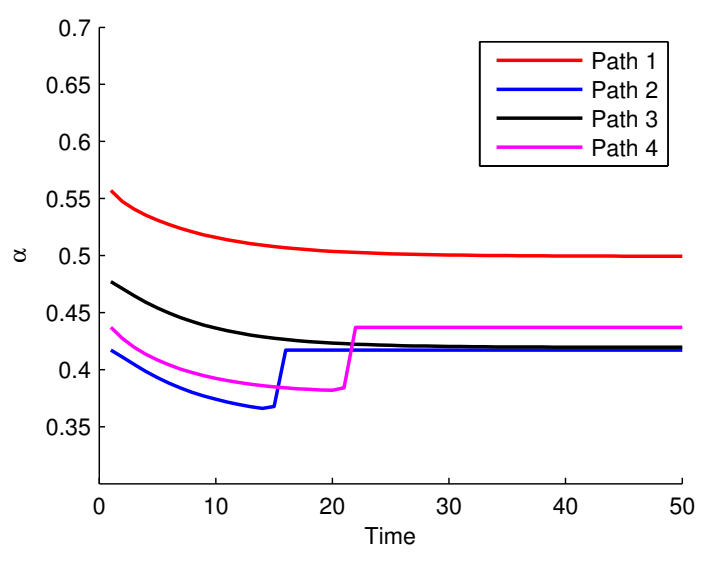

(b) Dynamical behavior of sentiment

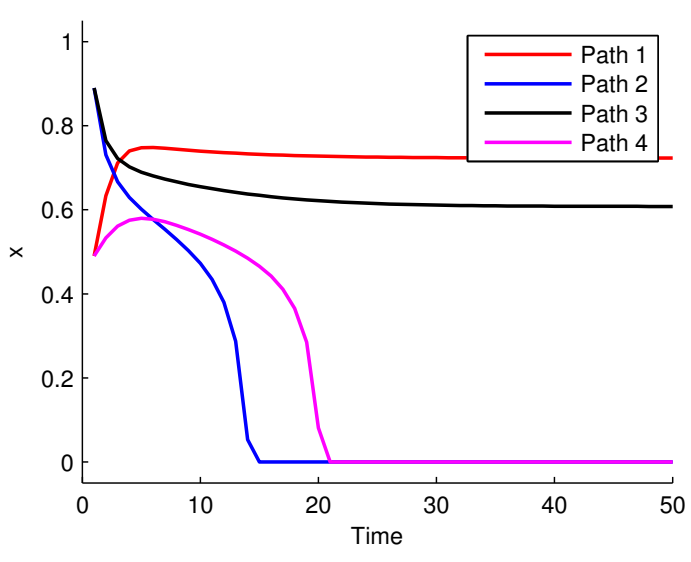

(c) Dynamical behavior of adoption rate

Figure 8: Four typical paths of $\left(\alpha^{t}, x^{t}\right)$ satisfying $\alpha^{1}<\alpha^{0}$. Points with a circle in subfigure (a) are the initial values. 


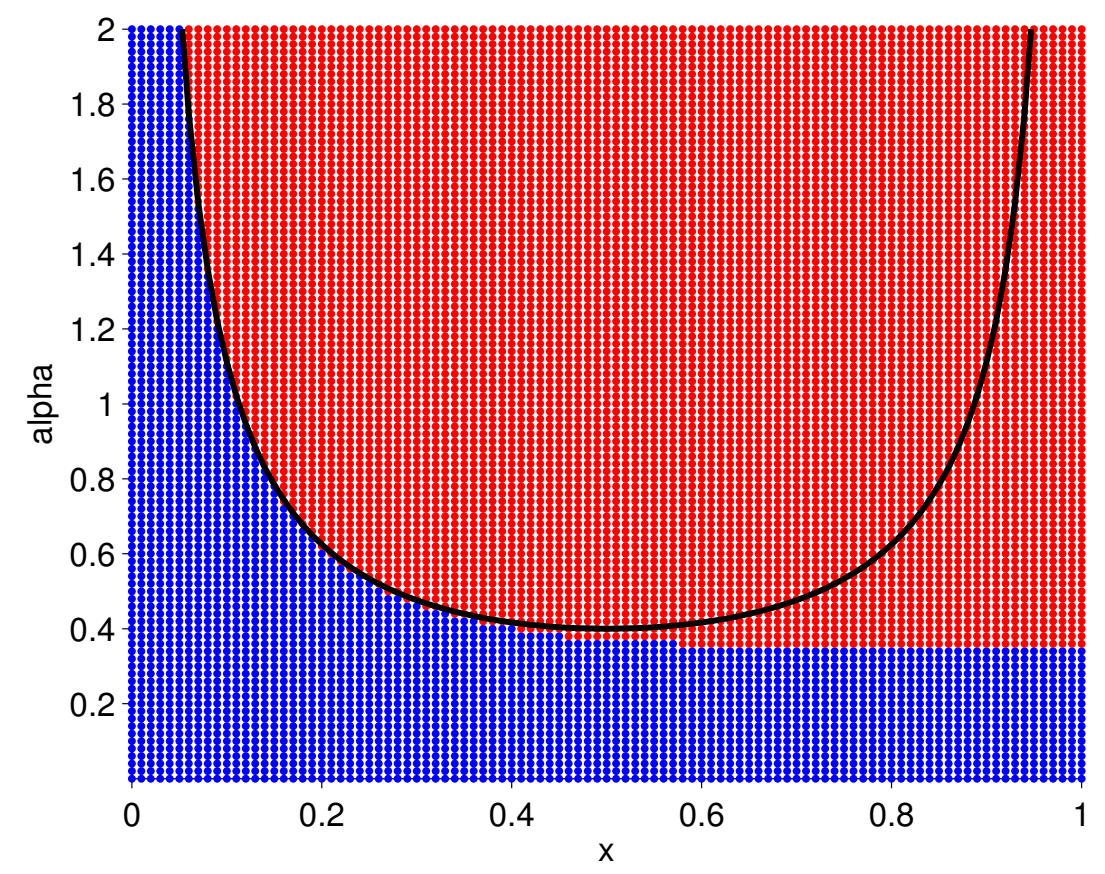

Figure 9: Basin of attraction corresponding to Figure 7: red initial points indicate convergence to non-trivial equilibrium on black curve, blue points convergence to a trivial equilibrium.

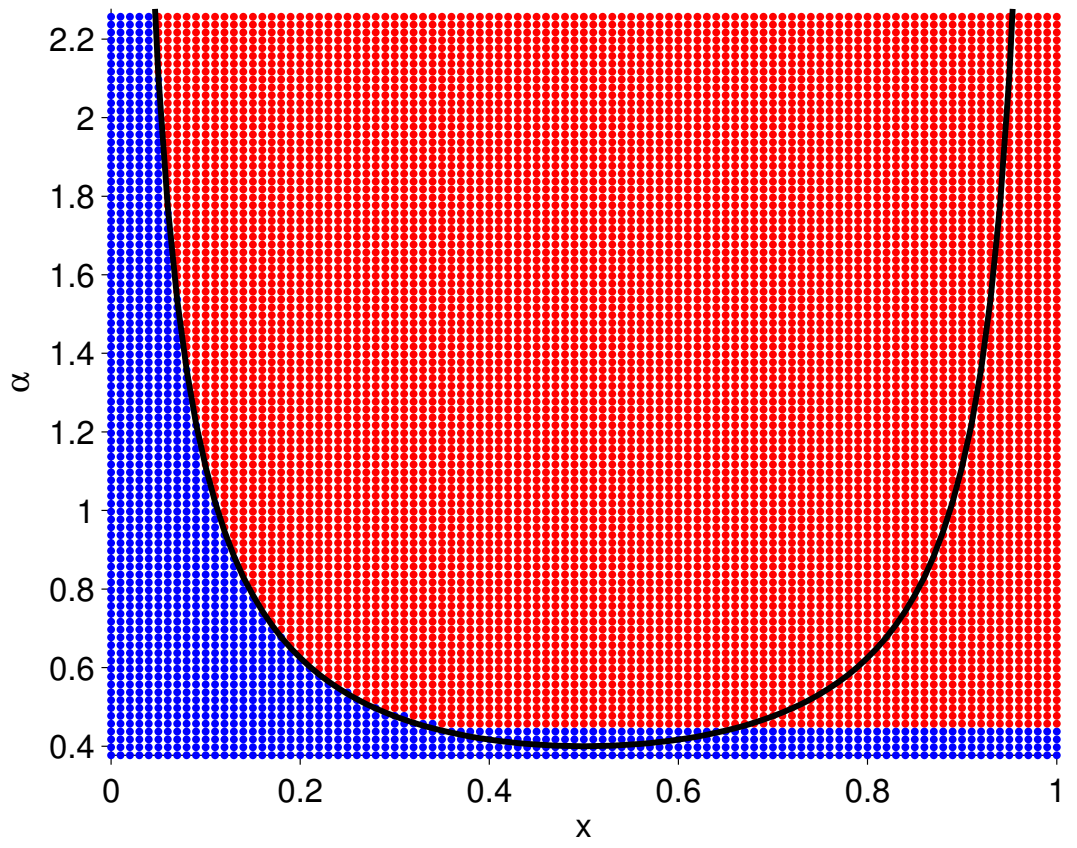

Figure 10: Basin of attraction corresponding to Figure 8. 
- Non-trivial attractors are located on the increasing part of the curve $\alpha=f(x)$.

- The domain $\mathbb{R}_{+} \times[0,1]$ of initial values can be divided into two areas with one of them containing initial values converging to non-trivial attractors, while the other leads to zero adoption rate in the long run. Part of the border between these two areas coincides with $\alpha=f(x)$.

\section{Concluding Remarks}

In this paper we proposed a network game model where the driving factor describing the inclination towards adopting a new behavior is endogenous. The learning dynamics about the inclinations was governed by the social network. There are four main insights that come out of the paper. First, collective adoption rate can display non-monotonic changes in behavior. This could help to understand 'sparks' of collective action, i.e. sudden change in behavior as if the collectivity was conducted. The explanation is that collective behavior is coupled to social learning, the invisible part, which governs by means of the social network. The second insight is that the convergence of individual inclinations is immediate provided the system starts from an average equilibrium. The third insight is that the definition of a critical mass is too short-sighted as its level depends on initial inclinations. The fourth insight is that despite the complexity of the model it is possible to derive equilibria in a closed form for network with low dimensionality. For networks with a larger number of possible degrees we have seen by simulation that collective behavior shows qualitatively different trajectories depending on the starting point. However, the long run behavior can be easily predicted since the model rules out cycling behavior. The fact that the network was given by a degree distribution instead of adjacency matrices allowed extensive simulation without running into storage problems.

Following Young [2009] we intended to keep the model general in order to keep it applicable to a broad range of problems. The dynamics is determined by a surprisingly small amount of parameter specifications. These are the social network and idiosynchratic costs, given by probability distributions, as well as an inertia parameter and a technology parameter which captures how the number of neighbors affects the individual benefits from adoption. ${ }^{13}$

\section{Appendix: Proofs of Propositions}

\section{Proof of Proposition 3}

We first assume $\tau_{d_{1}}=\tau_{d_{2}}$ and relax it later. Since $d \in\left\{d_{1}, d_{2}\right\}$, average inclination $\alpha$ in

\footnotetext{
${ }^{13}$ The network game is determined by $P, F,\left\{\tau_{d}\right\}$ and $\beta$.
} 
equilibria satisfies the following simplified equation

$$
\alpha=\widetilde{P}\left(d_{1}\right) \frac{\tau \alpha_{d_{1}}^{0}+d_{1} x \alpha}{\tau+d_{1} x}+\widetilde{P}\left(d_{2}\right) \frac{\tau \alpha_{d_{2}}^{0}+d_{2} x \alpha}{\tau+d_{2} x} .
$$

It follows that

$$
\begin{aligned}
\alpha= & \frac{\widetilde{P}\left(d_{1}\right)\left(\tau \alpha_{d_{1}}^{0}+d_{1} x \alpha\right)\left(\tau+d_{2} x\right)}{\left(\tau+d_{1} x\right)\left(\tau+d_{2} x\right)}+\frac{\widetilde{P}\left(d_{2}\right)\left(\tau \alpha_{d_{2}}^{0}+d_{2} x \alpha\right)\left(\tau+d_{1} x\right)}{\left(\tau+d_{1} x\right)\left(\tau+d_{2} x\right)} \\
= & \frac{\widetilde{P}\left(d_{1}\right)\left[\tau^{2} \alpha_{d_{1}}^{0}+\tau x\left(d_{1} \alpha+d_{2} \alpha_{d_{1}}^{0}\right)+x^{2} \alpha d_{1} d_{2}\right]}{\tau^{2}+\tau x\left(d_{1}+d_{2}\right)+x^{2} d_{1} d_{2}} \\
& \quad+\frac{\widetilde{P}\left(d_{2}\right)\left[\tau^{2} \alpha_{d_{2}}^{0}+\tau x\left(d_{2} \alpha+d_{1} \alpha_{d_{2}}^{0}\right)+x^{2} \alpha d_{1} d_{2}\right]}{\tau^{2}+\tau x\left(d_{1}+d_{2}\right)+x^{2} d_{1} d_{2}} \\
= & \frac{\tau^{2} \alpha^{0}+\tau x\left[\widetilde{P}\left(d_{1}\right)\left(d_{1} \alpha+d_{2} \alpha_{d_{1}}^{0}\right)+\widetilde{P}\left(d_{2}\right)\left(d_{2} \alpha+d_{1} \alpha_{d_{2}}^{0}\right)\right]+x^{2} \alpha d_{1} d_{2}}{\tau^{2}+\tau x\left(d_{1}+d_{2}\right)+x^{2} d_{1} d_{2}} \\
= & \frac{\tau^{2} \alpha^{0}+\tau x\left[\left\{\widetilde{P}\left(d_{1}\right) d_{1}+\widetilde{P}\left(d_{2}\right) d_{2}\right\} \alpha+\left\{\widetilde{P}\left(d_{1}\right) d_{2} \alpha_{d_{1}}^{0}+\widetilde{P}\left(d_{2}\right) d_{1} \alpha_{d_{2}}^{0}\right\}\right]+x^{2} \alpha d_{1} d_{2}}{\tau^{2}+\tau x\left(d_{1}+d_{2}\right)+x^{2} d_{1} d_{2}} \\
= & \alpha+E_{1}
\end{aligned}
$$

where

$$
\begin{aligned}
E_{1}= & \frac{\tau^{2}\left(\alpha^{0}-\alpha\right)}{\tau^{2}+\tau x\left(d_{1}+d_{2}\right)+x^{2} d_{1} d_{2}} \\
& +\frac{\tau x\left[\left\{\left(\widetilde{P}\left(d_{1}\right)-1\right) d_{1}+\left(\widetilde{P}\left(d_{2}\right)-1\right) d_{2}\right\} \alpha+\left\{\widetilde{P}\left(d_{1}\right) d_{2} \alpha_{d_{1}}^{0}+\widetilde{P}\left(d_{2}\right) d_{1} \alpha_{d_{2}}^{0}\right\}\right]}{\tau^{2}+\tau x\left(d_{1}+d_{2}\right)+x^{2} d_{1} d_{2}} \\
= & \frac{\tau^{2}\left(\alpha^{0}-\alpha\right)+\tau x\left[\left\{-\widetilde{P}\left(d_{2}\right) d_{1}-\widetilde{P}\left(d_{1}\right) d_{2}\right\} \alpha+\left\{\widetilde{P}\left(d_{1}\right) d_{2} \alpha_{d_{1}}^{0}+\widetilde{P}\left(d_{2}\right) d_{1} \alpha_{d_{2}}^{0}\right\}\right]}{\tau^{2}+\tau x\left(d_{1}+d_{2}\right)+x^{2} d_{1} d_{2}} \\
= & \frac{\tau^{2}\left(\alpha^{0}-\alpha\right)+\tau x\left[\widetilde{P}\left(d_{1}\right) d_{2}\left(\alpha_{d_{1}}^{0}-\alpha\right)+\widetilde{P}\left(d_{2}\right) d_{1}\left(\alpha_{d_{2}}^{0}-\alpha\right)\right]}{\tau^{2}+\tau x\left(d_{1}+d_{2}\right)+x^{2} d_{1} d_{2}} .
\end{aligned}
$$

Therefore it should be that $E_{1}=0$. Since $\tau>0, d_{1}>0, d_{2}>0$, and we are interested in non-trivial equilibria with $x \neq 0$, one has

$$
\begin{gathered}
\tau^{2}\left(\alpha^{0}-\alpha\right)+\tau x\left[\widetilde{P}\left(d_{1}\right) d_{2}\left(\alpha_{d_{1}}^{0}-\alpha\right)+\widetilde{P}\left(d_{2}\right) d_{1}\left(\alpha_{d_{2}}^{0}-\alpha\right)\right]=0 \\
\Leftrightarrow \quad \tau^{2} \alpha^{0}+\tau x\left[\widetilde{P}\left(d_{1}\right) d_{2} \alpha_{d_{1}}^{0}+\widetilde{P}\left(d_{2}\right) d_{1} \alpha_{d_{2}}^{0}\right]=\tau^{2} \alpha+\tau x\left[\widetilde{P}\left(d_{1}\right) d_{2}+\widetilde{P}\left(d_{2}\right) d_{1}\right] \alpha \\
\Leftrightarrow \quad \alpha=\frac{\tau^{2} \alpha^{0}+\tau x\left[\widetilde{P}\left(d_{1}\right) d_{2} \alpha_{d_{1}}^{0}+\widetilde{P}\left(d_{2}\right) d_{1} \alpha_{d_{2}}^{0}\right]}{\tau^{2}+\tau x\left[\widetilde{P}\left(d_{1}\right) d_{2}+\widetilde{P}\left(d_{2}\right) d_{1}\right]} \\
=\alpha^{0}+\frac{\tau x\left[\widetilde{P}\left(d_{1}\right) d_{2}\left(\alpha_{d_{1}}^{0}-\alpha^{0}\right)+\widetilde{P}\left(d_{2}\right) d_{1}\left(\alpha_{d_{2}}^{0}-\alpha^{0}\right)\right]}{\tau^{2}+\tau x\left[\widetilde{P}\left(d_{1}\right) d_{2}+\widetilde{P}\left(d_{2}\right) d_{1}\right]} .
\end{gathered}
$$

Here we use a small trick. $\alpha_{d_{1}}^{0}-\alpha^{0}$ can be rewritten as

$$
\begin{aligned}
\alpha_{d_{1}}^{0}-\alpha^{0} & =\alpha_{d_{1}}^{0}-\widetilde{P}\left(d_{1}\right) \alpha_{d_{1}}^{0}-\widetilde{P}\left(d_{2}\right) \alpha_{d_{2}}^{0}=\left\{1-\widetilde{P}\left(d_{1}\right)\right\} \alpha_{d_{1}}^{0}-\widetilde{P}\left(d_{2}\right) \alpha_{d_{2}}^{0} \\
& =\widetilde{P}\left(d_{2}\right)\left(\alpha_{d_{1}}^{0}-\alpha_{d_{2}}^{0}\right) .
\end{aligned}
$$


Similarly,

$$
\alpha_{d_{2}}^{0}-\alpha^{0}=\widetilde{P}\left(d_{1}\right)\left(\alpha_{d_{2}}^{0}-\alpha_{d_{1}}^{0}\right)
$$

Thus we have

$$
\begin{aligned}
\alpha & =\alpha^{0}+\frac{\tau x\left[\widetilde{P}\left(d_{1}\right) d_{2} \widetilde{P}\left(d_{2}\right)\left(\alpha_{d_{1}}^{0}-\alpha_{d_{2}}^{0}\right)+\widetilde{P}\left(d_{2}\right) d_{1} \widetilde{P}\left(d_{1}\right)\left(\alpha_{d_{2}}^{0}-\alpha_{d_{1}}^{0}\right)\right]}{\tau^{2}+\tau x\left[\widetilde{P}\left(d_{1}\right) d_{2}+\widetilde{P}\left(d_{2}\right) d_{1}\right]} \\
& =\alpha^{0}+\frac{\tau x\left(d_{2}-d_{1}\right) \widetilde{P}\left(d_{1}\right) \widetilde{P}\left(d_{2}\right)\left(\alpha_{d_{1}}^{0}-\alpha_{d_{2}}^{0}\right)}{\tau^{2}+\tau x\left[\widetilde{P}\left(d_{1}\right) d_{2}+\widetilde{P}\left(d_{2}\right) d_{1}\right]}
\end{aligned}
$$

If $\alpha$ is a fixed point, by setting $\alpha^{0}=\alpha$, it should hold that the second term of the above equation is 0 . This can only happen when $\alpha_{d_{1}}^{0}=\alpha_{d_{2}}^{0}$, which implies that each individual has the same initial inclination which does not change through our updating rule. This is equivalent to the one-dimensional process of $x^{t}$ with a constant $\alpha$.

For the case of $\tau_{d_{1}} \neq \tau_{d_{2}}$, the same procedure can be used. After some algebra we obtain an equation that needed to hold true for an equilibrium $\alpha$ as follows

$$
\alpha=\alpha^{0}+\frac{x\left(d_{2} \tau_{d_{1}}^{0}-d_{1} \tau_{d_{2}}^{0}\right) \widetilde{P}\left(d_{1}\right) \widetilde{P}\left(d_{2}\right)\left(\alpha_{d_{1}}^{0}-\alpha_{d_{2}}^{0}\right)}{\tau_{d_{1}}^{0} \tau_{d_{2}}^{0}+x\left[\widetilde{P}\left(d_{1}\right) d_{2} \tau_{d_{1}}^{0}+\widetilde{P}\left(d_{2}\right) d_{1} \tau_{d_{2}}^{0}\right]} .
$$

For equilibrium we need $\alpha=\alpha^{0}$, which implies an necessary condition that either $\alpha_{d_{1}}^{0}=$ $\alpha_{d_{2}}^{0}$ or $d_{2} \tau_{d_{1}}^{0}=d_{1} \tau_{d_{2}}^{0}$.

\section{Proof of Proposition 4}

The similar strategy as that in the proof of Proposition 3 is used, thus we only show an outline of analysis but ignore the details of algebra. In equilibrium, $\alpha$ must satisfy the following equation.

$$
\begin{aligned}
\alpha & =\sum_{d \in\left\{d_{1}, d_{2}, d_{3}\right\}} \widetilde{P}(d) \frac{\tau \alpha_{d}^{0}+d x \alpha}{\tau+d \alpha} \\
& =\widetilde{P}\left(d_{1}\right) \frac{\tau \alpha_{d_{1}}^{0}+d_{1} x \alpha}{\tau+d_{1} x}+\widetilde{P}\left(d_{2}\right) \frac{\tau \alpha_{d_{2}}^{0}+d_{2} x \alpha}{\tau+d_{2} x}+\widetilde{P}\left(d_{3}\right) \frac{\tau \alpha_{d_{3}}^{0}+d_{3} x \alpha}{\tau+d_{3} x}
\end{aligned}
$$

By reducing the fractions to a common denominator and after simplification, one has

$$
\alpha=\alpha+\frac{E_{2}^{N}}{E_{2}^{D}}
$$

where

$$
\begin{aligned}
& E_{2}^{N}=\tau^{3}\left(\alpha^{0}-\alpha\right) \\
& +\tau^{2} x\left[d_{1}\left\{\widetilde{P}\left(d_{2}\right)\left(\alpha_{d_{2}}^{0}-\alpha\right)+\widetilde{P}\left(d_{3}\right)\left(\alpha_{d_{3}}^{0}-\alpha\right)\right\}\right. \\
& +d_{2}\left\{\widetilde{P}\left(d_{3}\right)\left(\alpha_{d_{3}}^{0}-\alpha\right)+\widetilde{P}\left(d_{1}\right)\left(\alpha_{d_{1}}^{0}-\alpha\right)\right\} \\
& \left.+d_{3}\left\{\widetilde{P}\left(d_{1}\right)\left(\alpha_{d_{1}}^{0}-\alpha\right)+\widetilde{P}\left(d_{2}\right)\left(\alpha_{d_{2}}^{0}-\alpha\right)\right\}\right] \\
& +\tau x^{2}\left[d_{1} d_{2} \widetilde{P}\left(d_{3}\right)\left(\alpha_{d_{3}}^{0}-\alpha\right)+d_{2} d_{3} \widetilde{P}\left(d_{1}\right)\left(\alpha_{d_{1}}^{0}-\alpha\right)+d_{3} d_{1} \widetilde{P}\left(d_{2}\right)\left(\alpha_{d_{2}}^{0}-\alpha\right)\right]
\end{aligned}
$$


and

$$
E_{2}^{D}=\left(\tau+d_{1} x\right)\left(\tau+d_{2} x\right)\left(\tau+d_{3} x\right)>0
$$

Then we obtain $E_{2}^{N}=0$, and solving this equation for $\alpha$ yields $\alpha=\alpha^{0}+\frac{E_{3}^{N}}{E_{3}^{D}}$ where

$$
\begin{aligned}
E_{3}^{N}=\tau x[ & \left(\tau+d_{3} x\right)\left(d_{2}-d_{1}\right) \widetilde{P}\left(d_{1}\right) \widetilde{P}\left(d_{2}\right)\left(\alpha_{d_{1}}^{0}-\alpha_{d_{2}}^{0}\right) \\
& +\left(\tau+d_{1} x\right)\left(d_{3}-d_{2}\right) \widetilde{P}\left(d_{2}\right) \widetilde{P}\left(d_{3}\right)\left(\alpha_{d_{2}}^{0}-\alpha_{d_{3}}^{0}\right) \\
& \left.+\left(\tau+d_{2} x\right)\left(d_{3}-d_{1}\right) \widetilde{P}\left(d_{1}\right) \widetilde{P}\left(d_{3}\right)\left(\alpha_{d_{1}}^{0}-\alpha_{d_{3}}^{0}\right)\right]
\end{aligned}
$$

and

$$
\begin{aligned}
E_{3}^{D}=\tau^{3} & +\tau^{2} x\left[d_{1}\left\{\widetilde{P}\left(d_{2}\right)+\widetilde{P}\left(d_{3}\right)\right\}+d_{2}\left\{\widetilde{P}\left(d_{3}\right)+\widetilde{P}\left(d_{1}\right)\right\}+d_{3}\left\{\widetilde{P}\left(d_{1}\right)+\widetilde{P}\left(d_{2}\right)\right\}\right] \\
& +\tau x^{2}\left[d_{1} d_{2} \widetilde{P}\left(d_{3}\right)+d_{2} d_{3} \widetilde{P}\left(d_{1}\right)+d_{3} d_{1} \widetilde{P}\left(d_{2}\right)\right]>0
\end{aligned}
$$

In equilibrium it should hold that $E_{3}^{N}=0$. Unlike the 2-degrees case, here we have a sum of three terms which cannot be all positive or negative. So we need a careful discussion for different initial values of $\left\{\alpha_{d_{1}}^{0}, \alpha_{d_{2}}^{0}, \alpha_{d_{3}}^{0}\right\}$. Without loss of generality, we assume $d_{1}<d_{2}<d_{3}$. Distinguish the following cases where 1) $\alpha_{d}^{0}$ 's are equal, 2) two $\alpha_{d}^{0}$ 's are equal and 3) the $\alpha_{d}^{0}$ 's are different:

1) $\alpha_{d_{1}}^{0}=\alpha_{d_{2}}^{0}=\alpha_{d_{3}}^{0} \rightarrow E_{3}^{N}=0$.

2) $\alpha_{d_{1}}^{0}=\alpha_{d_{2}}^{0}<\alpha_{d_{3}}^{0} \rightarrow E_{3}^{N}<0$

$$
\begin{aligned}
& \alpha_{d_{1}}^{0}=\alpha_{d_{2}}^{0}>\alpha_{d_{3}}^{0} \rightarrow E_{3}^{N}>0 \\
& \alpha_{d_{1}}^{0}=\alpha_{d_{3}}^{0} \neq \alpha_{d_{2}}^{0} \rightarrow E_{3}^{N}=0 \text { if } \frac{\tau+d_{3} x}{\tau+d_{1} x}=\frac{\left(d_{3}-d_{2}\right) \widetilde{P}\left(d_{3}\right)}{\left(d_{2}-d_{1}\right) \widetilde{P}\left(d_{1}\right)} \\
& \alpha_{d_{2}}^{0}=\alpha_{d_{3}}^{0}<\alpha_{d_{1}}^{0} \rightarrow E_{3}^{N}>0 \\
& \alpha_{d_{2}}^{0}=\alpha_{d_{3}}^{0}>\alpha_{d_{1}}^{0} \rightarrow E_{3}^{N}<0
\end{aligned}
$$

3) $\alpha_{d_{1}}^{0}<\alpha_{d_{2}}^{0}<\alpha_{d_{3}}^{0} \rightarrow E_{3}^{N}<0$

$\alpha_{d_{1}}^{0}<\alpha_{d_{3}}^{0}<\alpha_{d_{2}}^{0} \rightarrow \alpha_{d}^{0}$ non-monotonic in $d$, discussion follows below $\alpha_{d_{2}}^{0}<\alpha_{d_{1}}^{0}<\alpha_{d_{3}}^{0} \rightarrow \alpha_{d}^{0}$ non-monotonic in $d$, discussion follows below $\alpha_{d_{2}}^{0}<\alpha_{d_{3}}^{0}<\alpha_{d_{1}}^{0} \rightarrow \alpha_{d}^{0}$ non-monotonic in $d$, discussion follows below $\alpha_{d_{3}}^{0}<\alpha_{d_{1}}^{0}<\alpha_{d_{2}}^{0} \rightarrow \alpha_{d}^{0}$ non-monotonic in $d$, discussion follows below $\alpha_{d_{3}}^{0}<\alpha_{d_{2}}^{0}<\alpha_{d_{1}}^{0} \rightarrow E_{3}^{N}>0$

As a conclusion, it is possible to have non-symmetric equilibria only if $\alpha_{d_{1}}^{0}=\alpha_{d_{3}}^{0} \neq \alpha_{d_{2}}^{0}$, or in cases that $\alpha_{d}^{0}$ 's are all different and not monotonic in $d$. Suppose $\alpha_{d_{1}}^{0}=\alpha_{d_{3}}^{0} \neq \alpha_{d_{2}}^{0}$, then we have

$$
\frac{\tau+d_{3} x}{\tau+d_{1} x}=\frac{\left(d_{3}-d_{2}\right) \widetilde{P}\left(d_{3}\right)}{\left(d_{2}-d_{1}\right) \widetilde{P}\left(d_{1}\right)}
$$


is a linear function of $x$, it is easy to find the condition for the existence of non-zero $x$ and thus we omit the calculation. The other cases, of non-monotonic $\alpha_{d}^{0}$, need to be discussed separately. We will focus on the case $\alpha_{d_{2}}^{0}<\alpha_{d_{1}}^{0}<\alpha_{d_{3}}^{0}$, since the story for other cases is similar. The equality $E_{3}^{N}=0$ holds if

$$
\begin{aligned}
& \left(\tau+d_{3} x\right)\left(d_{2}-d_{1}\right) \widetilde{P}\left(d_{1}\right) \widetilde{P}\left(d_{2}\right)\left(\alpha_{d_{1}}^{0}-\alpha_{d_{2}}^{0}\right) \\
& =\left(\tau+d_{1} x\right)\left(d_{3}-d_{2}\right) \widetilde{P}\left(d_{2}\right) \widetilde{P}\left(d_{3}\right)\left(\alpha_{d_{3}}^{0}-\alpha_{d_{2}}^{0}\right)+\left(\tau+d_{2} x\right)\left(d_{3}-d_{1}\right) \widetilde{P}\left(d_{1}\right) \widetilde{P}\left(d_{3}\right)\left(\alpha_{d_{3}}^{0}-\alpha_{d_{1}}^{0}\right) .
\end{aligned}
$$

Both sides of the above equation are larger then 0 . Solving this equation for $\alpha_{d_{3}}^{0}$ leads to $\alpha_{d_{3}}^{0}=C_{1} \alpha_{d_{1}}^{0}+C_{2} \alpha_{d_{2}}^{0}$ where

$$
\begin{aligned}
& C_{1}=\frac{\left(\tau+d_{3} x\right)\left(d_{2}-d_{1}\right) \widetilde{P}\left(d_{1}\right) \widetilde{P}\left(d_{2}\right)+\left(\tau+d_{2} x\right)\left(d_{3}-d_{1}\right) \widetilde{P}\left(d_{1}\right) \widetilde{P}\left(d_{3}\right)}{\left(\tau+d_{1} x\right)\left(d_{3}-d_{2}\right) \widetilde{P}\left(d_{2}\right) \widetilde{P}\left(d_{3}\right)+\left(\tau+d_{2} x\right)\left(d_{3}-d_{1}\right) \widetilde{P}\left(d_{1}\right) \widetilde{P}\left(d_{3}\right)}, \text { and } \\
& C_{2}=\frac{\left(\tau+d_{1} x\right)\left(d_{3}-d_{2}\right) \widetilde{P}\left(d_{2}\right) \widetilde{P}\left(d_{3}\right)-\left(\tau+d_{3} x\right)\left(d_{2}-d_{1}\right) \widetilde{P}\left(d_{1}\right) \widetilde{P}\left(d_{2}\right)}{\left(\tau+d_{1} x\right)\left(d_{3}-d_{2}\right) \widetilde{P}\left(d_{2}\right) \widetilde{P}\left(d_{3}\right)+\left(\tau+d_{2} x\right)\left(d_{3}-d_{1}\right) \widetilde{P}\left(d_{1}\right) \widetilde{P}\left(d_{3}\right)} .
\end{aligned}
$$

It is easy to see that $C_{1}+C_{2}=1, C_{1}>0$ and $C_{2}<1$. This means $\alpha_{d_{3}}^{0}$ is an affine combination of $\alpha_{d_{1}}^{0}$ and $\alpha_{d_{2}}^{0}$. Since $\alpha_{d_{2}}^{0}<\alpha_{d_{1}}^{0}<\alpha_{d_{3}}^{0}$, we need that $C_{1}>1$. Equivalently, we need that

$$
\begin{aligned}
& \left(\tau+d_{3} x\right)\left(d_{2}-d_{1}\right) \widetilde{P}\left(d_{1}\right) \widetilde{P}\left(d_{2}\right)>\left(\tau+d_{1} x\right)\left(d_{3}-d_{2}\right) \widetilde{P}\left(d_{2}\right) \widetilde{P}\left(d_{3}\right) \\
\Leftrightarrow & \frac{\tau+d_{3} x}{\tau+d_{1} x}>\frac{\left(d_{3}-d_{2}\right) \widetilde{P}\left(d_{3}\right)}{\left(d_{2}-d_{1}\right) \widetilde{P}\left(d_{1}\right)} .
\end{aligned}
$$

So (23) gives a necessary condition for existence of equilibria when $\alpha_{d_{2}}^{0}<\alpha_{d_{1}}^{0}<\alpha_{d_{3}}^{0}$. The next step is to find the valid corresponding non-zero fixed point value for $x$. Since $\alpha^{0}=\widetilde{P}\left(d_{1}\right) \alpha_{d_{1}}^{0}+\widetilde{P}\left(d_{2}\right) \alpha_{d_{2}}^{0}+\widetilde{P}\left(d_{3}\right) \alpha_{d_{3}}^{0}$, and from the relation $\alpha_{d_{3}}^{0}=C_{1} \alpha_{d_{1}}^{0}+C_{2} \alpha_{d_{2}}^{0}$, one has

$$
\alpha^{0}=\frac{A(x)}{A(x)+B(x)} \alpha_{d_{1}}^{0}+\frac{B(x)}{A(x)+B(x)} \alpha_{d_{2}}^{0}
$$

where $A(x)=\left(\tau+d_{2} x\right)\left(d_{3}-d_{1}\right) \widetilde{P}\left(d_{1}\right)$ and $B(x)=\left(\tau+d_{1} x\right)\left(d_{3}-d_{2}\right) \widetilde{P}\left(d_{2}\right)$. A non-trivial fixed point $(x, \alpha)$ satisfies equations

$$
\begin{aligned}
\alpha & =\frac{A(x)}{A(x)+B(x)} \alpha_{d_{1}}^{0}+\frac{B(x)}{A(x)+B(x)} \alpha_{d_{2}}^{0}, \\
x & =1-\frac{1}{b \alpha x},
\end{aligned}
$$

which boil down to

$$
A(x)+B(x)=b x(1-x) A(x) \alpha_{d_{1}}^{0}+b x(1-x) B(x) \alpha_{d_{2}}^{0} .
$$


Notice that the right-hand side of the equation (24) is 0 for $x=0$ and $x=1$ while for these values of $x$ the left-hand side is strictly positive. Then an equilibrium exists if and only if the mapping $h: x \mapsto A(x)+B(x)-b x(1-x) A(x) \alpha_{d_{1}}^{0}-b x(1-x) B(x) \alpha_{d_{2}}^{0}$ has a minimum value of at most 0 on $(0,1)$. This means that checking for equilibria involves only solving for critical points of $h$, i.e., solving a quadratic equation.

\section{Proof of Proposition 5}

For all $x^{t} \in(0,1]$, we first consider the case $\alpha^{t}>\frac{1}{b\left(1-x^{t}\right) x^{t}}$. It follows that

$$
\alpha^{t} \geq \frac{1}{b\left(1-x^{t}\right) x^{t}}>\frac{1}{b x^{t}} \quad \Rightarrow \quad x^{t}>\frac{1}{b \alpha^{t}} \quad \Leftrightarrow \quad \frac{1}{b \alpha^{t} x^{t}}<1 .
$$

From Equation (18), one then has

$$
x^{t+1}=1-\sum_{d} \min \left\{1, \frac{1}{b \alpha^{t} x^{t}}\right\} \widetilde{P}(d)=1-\sum_{d} \frac{1}{b \alpha^{t} x^{t}} \widetilde{P}(d)=1-\frac{1}{b \alpha^{t} x^{t}},
$$

which implies that

$$
x^{t+1}-x^{t}=1-x^{t}-\frac{1}{b \alpha^{t} x^{t}}>0 .
$$

The last inequality follows the condition $\alpha^{t}>\frac{1}{b\left(1-x^{t}\right) x^{t}}$ together with $\alpha^{t}>0$ and $x^{t}>0$. If $\alpha^{t}=\frac{1}{b\left(1-x^{t}\right) x^{t}}$, it is easy to see that $x^{t+1}-x^{t}=0$.

For $\alpha^{t}<\frac{1}{b\left(1-x^{t}\right) x^{t}}$, one has

$$
\alpha^{t}<\frac{1}{b\left(1-x^{t}\right) x^{t}} \quad \Leftrightarrow \quad 1-x^{t}<\frac{1}{b \alpha^{t} x^{t}} \quad \Leftrightarrow \quad 1-\frac{1}{b \alpha^{t} x^{t}}<x^{t} .
$$

Equation (18) again gives us

$$
\begin{aligned}
x^{t+1} & =1-\sum_{d} \min \left\{1, \frac{1}{b \alpha^{t} x^{t}}\right\} \widetilde{P}(d)=\sum_{d} \widetilde{P}(d)\left[1-\min \left\{1, \frac{1}{b \alpha^{t} x^{t}}\right\}\right] \\
& = \begin{cases}0 & \text { for } x^{t}<\frac{1}{b \alpha^{t}} \\
1-\frac{1}{b \alpha^{t} x^{t}} & \text { for } x^{t} \geq \frac{1}{b \alpha^{t}}\end{cases} \\
& <x^{t},
\end{aligned}
$$

which completes the proof.

\section{References}

M. Granovetter. Threshold Models of Collective Behavior. The American Journal of Sociology, 83(6):1420-1443, 1978. 
M. D. Groot. Optimal Statistical Decisions. Mc Graw Hill, 1970.

M. O. Jackson and L. Yariv. Diffusion on Social Networks. Économie Publique, 16:3-16, 2005.

M. O. Jackson and L. Yariv. Diffusion of Behavior and Equilibrium Properties in Network Games. American Economic Review, 97(2):92-98, 2007.

T. Kuran. Sparks and Prairie Fires: A Theory of Unanticipated Political Revolution. Public Choice, 61(1):41-74, 1989.

T. Kuran. The East European Revolution of 1989: Is it Surprising That We Were Surprised? The American Economic Review, 81(2):121-125, 1991.

M. Newman. Networks: An Introduction. Oxford University Press, 2010.

T. C. Schelling. Dynamic Models of Segregation. Journal of Mathematical; Sociology, 1: 143-186, 1971.

T. W. Valente. Network Models and Methods for Studying the Diffusion of Innovations. In Models and Methods in Social Network Analysis, pages 98-116. Cambridge University Press, 2005.

H. P. Young. Innovation Diffusion in Heterogeneous Populations: Contagion, Social Influence, and Social Learning. American Economic Review, 99(5):1899-1924, 2009. 